TRANSACTIONS OF THE

AMERICAN MATHEMATICAL SOCIETY

Volume 362, Number 6, June 2010, Pages 3125-3147

S 0002-9947(10)04811-7

Article electronically published on January 7, 2010

\title{
GRADED IDENTITIES OF MATRIX ALGEBRAS AND THE UNIVERSAL GRADED ALGEBRA
}

\author{
ELI ALJADEFF, DARRELL HAILE, AND MICHAEL NATAPOV
}

\begin{abstract}
We consider fine group gradings on the algebra $M_{n}(\mathbb{C})$ of $n$ by $n$ matrices over the complex numbers and the corresponding graded polynomial identities. Given a group $G$ and a fine $G$-grading on $M_{n}(\mathbb{C})$, we show that the $T$-ideal of graded identities is generated by a special type of identity, and, as a consequence, we solve the corresponding Specht problem for this case. Next we construct a universal algebra $U$ (depending on the group $G$ and the grading) in two different ways: one by means of polynomial identities and the other one by means of a generic two-cocycle (this parallels the classical constructions in the nongraded case). We show that a suitable central localization of $U$ is Azumaya over its center and moreover, its homomorphic images are precisely the $G$-graded forms of $M_{n}(\mathbb{C})$. Finally, we consider the ring of central quotients of $U$ which is a central simple algebra over the field of quotients of the center of $U$. Using earlier results of the authors we show that this is a division algebra if and only if the group $G$ is one of a very explicit (and short) list of nilpotent groups. It follows that for groups not on this list, one can find a nonidentity graded polynomial such that its power is a graded identity. We illustrate this phenomenon with an explicit example.
\end{abstract}

\section{INTRODUCTION}

In the last decade, group gradings and graded identities of finite dimensional central simple algebras have been an active area of research. We refer the reader to Bahturin, et al. 8], 9] and [7. There are two basic kinds of group grading, elementary and fine. It was proved by Bahturin and Zaicev $[9]$ that any group grading of $A=M_{n}(\mathbb{C})$ is given by a certain "composition" of an elementary grading and a fine grading. In 7], Bahturin and Drensky consider graded polynomial identities and the codimension growth of elementary and fine gradings on $A$. In particular they provide bases for the $G$-graded identities on $A$ in two cases: (a) $G$ is any group, the $G$-grading is elementary and the identity component $A_{e}$ coincides with the subalgebra of diagonal matrices; (b) $G$ is abelian and the $G$-grading is fine. In this paper we are concerned with fine gradings and their graded identities on $A$, where $G$ is arbitrary.

Let $R$ be a simple algebra, finite dimensional over its center $k$ and let $G$ be any group. We say that $R$ is fine graded by $G$ if $R \cong \bigoplus_{g \in G} R_{g}$ is a grading and $\operatorname{dim}_{k}\left(R_{g}\right) \leq 1$. Thus any component is either 0 or isomorphic to $k$ as a $k$-vector space. It is easy to show that $\operatorname{Supp}(R)$, the subset of elements of $G$ for which $R_{g}$ is

Received by the editors October 26, 2007 and, in revised form, April 22, 2008.

2000 Mathematics Subject Classification. Primary 16W50, 16R10, 16R50; Secondary 16S35, $16 \mathrm{~K} 20$.

(C)2010 American Mathematical Society Reverts to public domain 28 years from publication 
not 0 , is a finite subgroup of $G$. Moreover $R$ is strongly graded by $\operatorname{Supp}(R)$, namely $R_{g} R_{h}=R_{g h}$ for every $g, h \in \operatorname{Supp}(R)$. Since every group $\Gamma$ containing $\operatorname{Supp}(R)$ provides a fine grading of $R$ (just by putting $R_{g}=0$ for $g$ outside $\operatorname{Supp}(R)$ ), we will restrict our attention to the case where $G=\operatorname{Supp}(R)$. In this case it has been shown (see Bahturin and Zaicev [9]) that the existence of such a grading is equivalent to $R$ being isomorphic to a twisted group algebra $k^{c} G$, where $[c]$ is an element in $H^{2}\left(G, k^{\times}\right)$and $G$ acts trivially on $k^{\times}$.

A group $G$ is said to be of central type if it admits a cocycle $c,[c] \in H^{2}\left(G, \mathbb{C}^{\times}\right)$, for which the twisted group algebra $\mathbb{C}^{c} G$ is central simple over $\mathbb{C}$. By definition such a cocycle $c$ is called nondegenerate. As we have just seen, fine gradings arise from such groups and cocycles. Groups of central type appear in the theory of projective representations of finite groups and in the classification of finite dimensional Hopf algebras. Using the classification of finite simple groups, Howlett and Isaacs [14] proved that any group of central type is solvable.

Given a (fine) $G$-grading on $M_{n}(\mathbb{C})$ we consider the set of graded identities: let $k$ be a subfield of $\mathbb{C}$ and let $\Omega=\left\{x_{i g}: i \in \mathbb{N}, g \in G\right\}$ be a set of indeterminates. Let $\Sigma(k)=k\langle\Omega\rangle$ be the noncommutative free algebra generated by $\Omega$ over $k$. A polynomial $p\left(x_{i g}\right) \in \Sigma(k)$ is a graded identity of $M_{n}(\mathbb{C})$ if the polynomial vanishes upon every substitution of the indeterminates $x_{i g}$ by elements of degree $g$ in $M_{n}(\mathbb{C})$. It is clear that the set of graded identities is an ideal of $\Sigma(k)$. Furthermore, it is a graded $T$-ideal. Recall that an ideal (in $\Sigma(k))$ is a graded $T$-ideal if it is closed under all $G$-graded endomorphisms of $\Sigma(k)$. In section two we show that all graded identities are already defined over a certain finite cyclotomic field extension $\mathbb{Q}(\mu)$ of $\mathbb{Q}$. Moreover $\mathbb{Q}(\mu)$ is minimal and unique with that property. We refer to $\mathbb{Q}(\mu)$ as the field of definition of the graded identities. We consider the free algebra $\Sigma(\mathbb{Q}(\mu))$ and denote by $T(\mathbb{Q}(\mu))$ the $T$-ideal of graded identities of $M_{n}(\mathbb{C})$ in $\Sigma(\mathbb{Q}(\mu))$. We introduce a set of special graded identities, which we call "elementary". We show that a certain finite subset of the set of elementary identities generates $T(\mathbb{Q}(\mu))$. In particular $T(\mathbb{Q}(\mu))$ is finitely generated as a $T$-graded ideal.

In section three we examine the algebra $U_{G}=\Sigma(\mathbb{Q}(\mu)) / T(\mathbb{Q}(\mu))$, which we call the universal $G$-graded algebra. First we introduce another algebra analogous to the ring of generic matrices in the classical theory. We show this algebra is isomorphic to $U_{G}$ and thus are able to prove that the center $Z=Z\left(U_{G}\right)$ is a domain and that if $F$ denotes the field of fractions of $Z$, then the algebra $Q\left(U_{G}\right)=F \otimes_{Z} U_{G}$ is an $F$-central simple algebra of dimension equal to the order of $G$. In particular $U_{G}$ is a prime ring. Moreover we show there is a certain multiplicatively closed subset $M$ in $Z$ such that the central localization $M^{-1} U_{G}$ is an Azumaya algebra over its center $S=M^{-1} Z$. The simple images of this Azumaya algebra are the graded forms of $M_{n}(\mathbb{C})$, that is, the $G$-graded central simple $L$-algebras $B$ such that $B \otimes_{L} \mathbb{C}$ is isomorphic as a graded algebra to $M_{n}(\mathbb{C})$, where $L$ varies over all subfields of $\mathbb{C}$. We also give quite explicit determinations of $S$ and $F$.

Note that these algebras depend on the given grading on $M_{n}(\mathbb{C})$, and so we should write $U_{G, c}, M^{-1} U_{G, c}$ and $Q\left(U_{G, c}\right)$, where $c$ is the given nondegenerate twococycle. We will omit the $c$ except in those cases where we need to emphasize the particular grading.

The constructions of the universal algebra presented in this section, namely by means of graded identities or by means of a generic two-cocycle, can be extended to the general context of Hopf Galois extensions, where the group algebra is replaced 
by an arbitrary Hopf algebra (see Aljadeff and Kassel [4). This extension establishes a bridge between the results here and classification problems in the theory of quantum groups and noncommutative geometry.

Unlike the case of classical polynomial identities, the central simple algebra $Q\left(U_{G}\right)$ is not necessarily a division algebra. Based on earlier work of the authors [2], we in fact show that $Q\left(U_{G, c}\right)$ is a division algebra if and only if the group $G$ belongs to a certain explicit list $\Lambda$ of groups. This is independent of the cocycle $c$, a fact we will return to below. The list $\Lambda$ consists of a very special family of nilpotent groups. Roughly speaking these are the nilpotent groups for which each Sylow $p$-subgroup is the direct product of an abelian group of the form $A \times A$ (said to be of symmetric type) and possibly a unique nonabelian group of the form $C_{p^{n}} \ltimes C_{p^{n}}$. For $p=2$ an extra family of nonabelian groups can occur, namely $\left(C_{2} \times C_{2^{n-1}}\right) \ltimes C_{2^{n}}$. The precise definition of $\Lambda$ is given in the last section.

The main result of the final section is that for every group $G$ on the list $\Lambda$ the automorphism group of $G$ acts transitively on the cohomology classes represented by nondegenerate two-cocycles. It follows that the algebras $Q\left(U_{G, c}\right)$ are all isomorphic for a fixed $G$ (but not graded isomorphic).

For a group $G$ of central type, let $\operatorname{ind}(G)$ denote the maximum over all nondegenerate cocycles $c$ of the indices of the simple algebras $Q\left(U_{G, c}\right)$. We have just seen that if $G$ is not on the list, then $\operatorname{ind}(G)$ is strictly less than the order of $G$. In a forthcoming paper, Aljadeff and Natapov [5], it is shown that in fact the groups on the list are the only groups responsible for the index. The precise result is stated in the last section (Theorem 21). It follows from this theorem that if $G$ is a $p$-group of central type, then $\operatorname{ind}(G) \leq \max \left(\operatorname{ord}(H)^{1 / 2}\right)$, where the maximum is taken over all $p$-groups $H$ on the list that are subquotients of $G$.

If $Q\left(U_{G}\right)$ is not a division algebra, there are graded identities over the field of definition that are the product of nonidentities. We present an explicit example of a grading on $M_{6}(\mathbb{C})$ (for the group $G=S_{3} \ltimes C_{6}$ ) for which there is a graded identity over the field of definition that is the cube of a nonidentity.

Even in the case where $Q\left(U_{G}\right)$ is a division algebra it is possible that this universal algebra does not remain a division algebra under extension of the coefficient field. In other words the algebra $Q\left(U_{G}\right) \otimes_{\mathbb{Q}(\mu)} \mathbb{C}$ may not be a division algebra. This means that there can be gradings on matrices for which the algebra $Q\left(U_{G}\right)$ is a division algebra and yet there are graded identities over $\mathbb{C}$ that are the products of nonidentities. In the last section we compute explicitly the index of this extended algebra $Q\left(U_{G}\right) \otimes_{\mathbb{Q}(\mu)} \mathbb{C}$ for groups $G$ on the list $\Lambda$.

\section{Graded identities}

Let $M_{n}(\mathbb{C})$ have a fine grading by the finite group $G$. In this section we investigate the $T$-ideal of $G$-graded identities on $M_{n}(\mathbb{C})$. As we have seen in the introduction the existence of such a grading implies that $G$ is of central type and that there is a nondegenerate cocycle $c$ such that $M_{n}(\mathbb{C})$ is isomorphic to the twisted group algebra $\mathbb{C}^{c} G$. We want to make this more precise. Let $A=M_{n}(\mathbb{C})$. The $G$-grading induces a decomposition $A \cong \bigoplus A_{g}$, where each homogeneous component is 1-dimensional over $\mathbb{C}$. Every homogeneous component $A_{g}$ is spanned by an invertible element $u_{g}$ (which we also fix from now on), and hence any element in $A_{g}$ is given by $\lambda_{g} u_{g}$, where $\lambda_{g} \in \mathbb{C}$. The multiplication is given by $u_{g} u_{h}=c(g, h) u_{g h}$, where $c(g, h) \in \mathbb{C}^{\times}$is a two-cocycle. We let $[c] \in H^{2}\left(G, \mathbb{C}^{\times}\right)$be the corresponding 
cohomology class. In this way we identify $A$ (as a $G$-graded algebra) with the twisted group algebra $\mathbb{C}^{c} G$. Replacing $c$ by a cohomologous cocycle $c^{\prime}$ produces a twisted group algebra $\mathbb{C}^{c^{\prime}} G$ that is isomorphic as a $G$-graded algebra to $A$.

We want to obtain information about the $T$-ideal of identities of $M_{n}(\mathbb{C})$. We begin with a special family of identities. As in the introduction we let $\Sigma(\mathbb{C})=\mathbb{C}\langle\Omega\rangle$ denote the free algebra generated by $\Omega$ over $\mathbb{C}$, where $\Omega=\left\{x_{i g}: i \in \mathbb{N}, g \in G\right\}$.

Let $Z_{1}=x_{r_{1} g_{1}} x_{r_{2} g_{2}} \cdots x_{r_{k} g_{k}}$ and $Z_{2}=x_{s_{1} h_{1}} x_{s_{2} h_{2}} \cdots x_{s_{l} h_{l}}$ be two monomials in $\Sigma(\mathbb{C})$. We say $Z_{1}$ and $Z_{2}$ are congruent if the following three properties are satisfied:

a) $g_{1} \cdots g_{k}=h_{1} \cdots h_{l}$

b) $k=l$ (equal length);

c) there exists an element $\pi \in \operatorname{Sym}(k)$ such that for $1 \leq i \leq k, s_{i}=r_{\pi(i)}$ and $h_{i}=g_{\pi(i)}$.

We say that $Z_{1}$ and $Z_{2}$ are weakly congruent if they satisfy condition (a). Clearly congruence and weak congruence are equivalence relations.

Let $c\left(g_{1}, g_{2}, \ldots, g_{k}\right)$ be the element in $\mathbb{C}^{\times}$determined by $u_{g_{1}} u_{g_{2}} \cdots u_{g_{k}}=$ $c\left(g_{1}, \ldots, g_{k}\right) u_{g_{1} \cdots g_{k}}$ in $\mathbb{C}^{c} G$. For any two congruent monomials $Z_{1}=x_{r_{1} g_{1}} x_{r_{2} g_{2}} \ldots$ $x_{r_{k} g_{k}}$ and $Z_{2}=x_{r_{\pi(1)} g_{\pi(1)}} x_{r_{\pi(2)} g_{\pi(2)}} \cdots x_{r_{\pi(k)} g_{\pi(k)}}$ consider the binomial

$$
B=B\left(Z_{1}, Z_{2}\right)=Z_{1}-\frac{c\left(g_{1}, \ldots, g_{k}\right)}{c\left(g_{\pi(1)}, \ldots, g_{\pi(k)}\right)} Z_{2} .
$$

It is easy to see that $B$ is a graded identity. We will call it an elementary identity. We refer to $c(B)=\frac{c\left(g_{1}, \ldots, g_{k}\right)}{c\left(g_{\pi(1)}, \ldots, g_{\pi(k)}\right)}$ in $\mathbb{C}^{\times}$as the coefficient of the elementary identity $B$.

This element $c(B)$ can be understood homologically. Let $F=F(\Omega)$ be the free group generated by $\Omega=\left\{x_{i g}: i \in \mathbb{N}, g \in G\right\}$. Let $\nu: F \longrightarrow G\left(x_{i g} \longmapsto g\right)$ be the natural map and $R=\operatorname{ker}(\nu)$. If $\pi \in \operatorname{Sym}(k)$ and $Z_{1}=x_{r_{1} g_{1}} x_{r_{2} g_{2}} \cdots x_{r_{k} g_{k}}, Z_{2}=$ $x_{r_{\pi(1)} g_{\pi(1)}} x_{r_{\pi(2)} g_{\pi(2)}} \cdots x_{r_{\pi(k)} g_{\pi(k)}}$ are congruent, then the element $z=Z_{1}\left(Z_{2}\right)^{-1}$ lies in $[F, F] \cap R$. By the Hopf formula, the Schur multiplier $M(G)$ of $G$ is given by $M(G)=[F, F] \cap R /[F, R]$ and so we may consider the element $\bar{z} \in M(G)$. Moreover the Universal Coefficient Theorem provides an isomorphism $\phi$ from $H^{2}\left(G, \mathbb{C}^{\times}\right)$to $\operatorname{Hom}\left(M(G), \mathbb{C}^{\times}\right)$,

Proposition 1. Let the notation be as given above.

(a) The element $c(B)$ is independent of the representing cocycle $c$ of $[c]$.

(b) If $z=Z_{1}\left(Z_{2}\right)^{-1}$ is as given above, then $c(B)=\phi([c])(\bar{z})$.

(c) Each $c(B)$ is a root of unity, in fact $c(B)^{\operatorname{ord}(G)}=1$.

(d) If $J$ denotes the set of all elementary identities, then the set $\mu=\{c(B) \in$ $\left.\mathbb{C}^{\times}: B \in J\right\}$ is finite.

Proof. Statement (a) is clear. For the proof of (b) we consider the central extension

$$
\{1\} \rightarrow R /[F, R] \rightarrow F /[F, R] \rightarrow G \rightarrow\{1\}
$$

induced by the map $\nu$ and let

$$
\{1\} \rightarrow \mathbb{C}^{\times} \rightarrow \Gamma \rightarrow G \rightarrow\{1\}
$$

be the central extension that corresponds to the cocycle $c$ (i.e. $\left\{u_{g}\right\}_{g \in G}$ is a set of representatives in $\Gamma$ and $\left.u_{g} u_{h}=c(g, h) u_{g h}\right)$. It is easy to see that the map $\gamma: F /[F, R] \rightarrow \Gamma$ given by $\gamma\left(x_{i g}\right)=u_{g}$ induces a map of extensions, and it is well known that its restriction $\gamma:[F, F] \cap R /[F, R]=M(G) \rightarrow \mathbb{C}^{\times}$is independent of the presentation and corresponds to $[c]$ by means of the Universal Coefficient Theorem. 
That is, $\phi([c])=\gamma$. It follows that $\phi([c])(\bar{z})=\gamma(\bar{z})=u_{g_{1}} \cdots u_{g_{r}}\left(u_{g_{\pi(1)}} \cdots u_{g_{\pi(r)}}\right)^{-1}$ $=\frac{c\left(g_{1}, \ldots, g_{k}\right)}{c\left(g_{\pi(1)}, \ldots, g_{\pi(k)}\right)}=c(B)$, as desired.

Statements (c) and (d) are direct consequences of (b). This completes the proof of the proposition.

From the proposition it follows that the set $J$ is defined over $Q(\mu)$. Our next task is to show that the $T$-ideal of graded identities $T(\mathbb{C})$ is spanned by $J$ and that $Q(\mu)$ is the field of definition for the graded identities. We will need the following terminology:

Let $k$ be a subfield of $\mathbb{C}$ and let $p\left(x_{r_{i} g_{i}}\right)=\sum \lambda_{r_{1} g_{1}, r_{2} g_{2}, \ldots, r_{k} g_{k}} x_{r_{1} g_{1}} x_{r_{2} g_{2}} \cdots x_{r_{k} g_{k}}$ be a polynomial in $\Sigma(k)(\subseteq \Sigma(\mathbb{C}))$. We say that $p\left(x_{r_{i} g_{i}}\right)$ is reduced if the monomials $x_{r_{1} g_{1}} x_{r_{2} g_{2}} \cdots x_{r_{k} g_{k}}$ are all different. If $p\left(x_{r_{i} g_{i}}\right)$ is reduced, we say that it is weakly homogeneous if its monomials are all weakly congruent and we say $p\left(x_{r_{i} g_{i}}\right)$ is homogeneous if its monomials are all congruent. It is clear that any reduced polynomial can be written uniquely as the sum of its weakly homogeneous components and that each weakly homogeneous component can be written uniquely as the sum of its homogeneous components.

Proposition 2. If $p\left(x_{r_{i} g_{i}}\right)$ is a graded identity, then its homogeneous components are graded identities as well. Moreover, any graded identity is a linear combination of elementary identities.

Proof. Let us show first that its weakly homogeneous components are graded identities. Indeed, the replacement of $x_{r_{i} g_{i}}$ by $u_{g_{i}} \in \mathbb{C}^{c} G$ maps weakly homogeneous components of $p\left(x_{r_{i} g_{i}}\right)$ to different homogeneous components (in the graded decomposition of $\mathbb{C}^{c} G$ ). Since these are linearly independent, they all must be 0 . Next, assume that we have at least two homogeneous components in a weakly homogeneous component, which we denote by $\Pi$. Then there exists an indeterminate $y=x_{r_{j} g_{j}}$ which appears with different multiplicities in (at least) two different monomials. Let $s \geq 1$ be the maximal multiplicity. We decompose $\Pi$ into (at most) $s+1$ components $U_{s}, \ldots, U_{0}$, where $U_{i}$ consists of the monomials of $\Pi$ that contain $y$ with multiplicity $i$. Of course, by induction, the result will follow if we show that $U_{s}$ is a graded identity. Suppose not. Then there is a substitution which does not annihilate $U_{s}$. Note that since $\Pi$ is weakly homogeneous the images of all components are multiples of $u_{g}$ in $\mathbb{C}^{c} G$, and hence there is an evaluation that maps $U_{i}$ to $\lambda_{i} u_{g}$ with $\lambda_{s}$ not zero. We may multiply the evaluation for $y$ by a central indeterminate $z$. Then we get a nonzero polynomial in $z$, whose coefficients are $\lambda_{i}$ and for any evaluation of $z$ we get zero. This is of course impossible in a field of characteristic zero.

To complete the proof of the proposition it suffices to show that every homogeneous identity is a linear combination of elementary identities. But this is clear since any two monomials which are congruent determine an elementary identity (and monomials are not identities).

Proposition 3. If $L$ is a subfield of $\mathbb{C}$ such that $\mathbb{Q}(\mu) \subseteq L \subseteq \mathbb{C}$, then the set $J$ spans $T(L)$ over $L$. Conversely, if $L \subseteq \mathbb{C}$ is a field of definition for $T(\mathbb{C})$, that is, $T(\mathbb{C})=T(L) \otimes_{L} \mathbb{C}$, then $L$ contains $\mathbb{Q}(\mu)$.

Proof. Fix a monomial $Z=x_{r_{1} g_{1}} x_{r_{2} g_{2}} \cdots x_{r_{k} g_{k}}$. Let $Z=Z_{1}, Z_{2}, \ldots, Z_{d}$ be the distinct monomials that are congruent to $Z$ and let $W$ be the $d$-dimensional $\mathbb{C}$ vector space spanned by these monomials. Each $Z_{i}$, for $1 \leq i<d$, determines an 
elementary identity $Z_{i}-c_{i} Z_{d}$, and these identities form a basis for the subspace $Y$ of graded identities in $W$. In particular the dimension of $Y$ over $\mathbb{C}$ is $d-1$. Furthermore if $\gamma=\left\{c_{1}, \ldots, c_{d-1}\right\}$, then clearly $Y$ is defined over $\mathbb{Q}(\gamma)$. But $\gamma \subseteq \mu$ and so it follows that $T(\mathbb{C})$ is defined over $\mathbb{Q}(\mu)$ and hence over any field $L$ that contains $\mathbb{Q}(\mu)$.

For the converse let $W_{L}$ denote the $L$-span of the monomials $Z_{1}, \ldots, Z_{d}$ and let $Y_{L}=Y \cap W_{L}$. We claim that if $L$ is a field of definition for $Y$ (that is, $Y=Y_{L} \otimes_{L} \mathbb{C}$ ), then $L \supseteq \mathbb{Q}(\gamma)$. Because $Z$ is arbitrary, it will follow from the claim that $L$ contains $\mu$. To prove the claim, let $f_{1}, \ldots, f_{d-1}$ be a basis of $Y_{L}$ over $L$. Express $f_{1}, \ldots, f_{d-1}$ using the monomials $Z_{i}$. The coefficient matrix is a $(d-1) \times d$ matrix over $L$. This matrix may be row reduced to the normal form $\left(I_{d-1}, C^{\prime}\right)$, where $C^{\prime}$ is a $(d-1) \times 1$ matrix over $L$. The column vector $C^{\prime}$ is uniquely determined. In other words there are unique scalars $a_{i}$ in $L$, for $1 \leq i<d$, such that the vectors $Z_{i}-a_{i} Z_{d}$ form a basis for $Y_{L}$ over $L$. On the other hand the identities $Z_{i}-c_{i} Z_{d}$ form a basis of $Y_{L(\gamma)}$ over $L(\gamma)$. It follows that $a_{i}=c_{i} \in L$ for all $i$.

We now show that the $T$-ideal of graded identities is finitely generated. Let $n=\operatorname{ord}(G)$. Consider the set $V=\left\{x_{i g}: 1 \leq i \leq n, g \in G\right\}$ of indeterminates and let $E$ be the set of elementary identities of length $\leq n$ (that is, where the monomials are of length $\leq n$ ) and such that its indeterminates are elements of $V$. Clearly $E$ is a finite set.

Theorem 4. The ideal of graded identities is generated as a T-ideal by E. In particular the ideal is finitely generated as a T-ideal.

Proof. Denote by $I^{\prime}$ the $T$-ideal generated by $E$. We will show that $I^{\prime}$ contains all graded identities. Clearly it is enough to show that $I^{\prime}$ contains all elementary identities. Let $B=x_{r_{1} g_{1}} x_{r_{2} g_{2}} \cdots x_{r_{k} g_{k}}-c(B) x_{r_{\pi(1)} g_{\pi(1)}} x_{r_{\pi(2)} g_{\pi(2)}} \cdots x_{r_{\pi(k)} g_{\pi(k)}}$ be an elementary identity of length $k$. Clearly, if $k \leq n$, then $B$ is in $I^{\prime}$. We assume therefore that $k>n$ and proceed by induction on $k$. The first observation is that if there exist $i, t$, with $1 \leq i, t \leq k-1, i=\pi(t)$ and $i+1=\pi(t+1)$, then we can reduce the length of the word: We let $g=g_{i} g_{i+1}$ and replace $x_{r_{i} g_{i}} x_{r_{i+1} g_{i+1}}$ by $x_{r g}$, where $1 \leq r \leq n$ and $x_{r g}$ does not appear in $B$. The resulting identity (one has to check that the coefficient is right) has shorter length, and so we may assume this identity is in $I^{\prime}$. But we can obtain the longer one from the shorter one by the substitution of $x_{r_{i} g_{i}} x_{r_{i+1} g_{i+1}}$ for $x_{r g}$, so we are done in this case.

Next, note that since $k>n$, the pigeonhole principle applied to the expressions $g_{1} \cdots g_{s}, s=1, \ldots, k$ shows that there are integers $1 \leq i<j \leq n$ such that $g_{i} g_{i+1} \cdots g_{j}=1$. Observe also that if $\gamma$ is a cyclic permutation of the numbers $i, i+1, \ldots, j$, then we have $g_{\gamma(i)} \cdots g_{\gamma(j)}=1$. That means that if we replace the string $x_{r_{i} g_{i}} x_{r_{i+1} g_{i+1}} \cdots x_{r_{j} g_{j}}$ by a cyclic permutation of the variables in the first monomial and leave the second monomial alone, we do not change our identity modulo $I^{\prime}$. Also for all $g \in G$, we have $g g_{i} g_{i+1} \cdots g_{j}=g_{i} g_{i+1} \cdots g_{j} g$. This means that modulo $I^{\prime}$ we can move the string $x_{r_{i} g_{i}} x_{r_{i+1} g_{i+1}} \cdots x_{r_{j} g_{j}}$ anywhere in the monomial. So, combining these two things we can cyclically move the substring and move it anywhere in the first monomial. But by doing these moves we can arrange it so that some two variables that are next to each other in the second monomial are also next to each other (in the same order) in the first monomial. This reduces us to the first case. 


\section{The Universal AlgEBRA}

In this section we determine the basic structural properties of the universal $G$ graded algebra $U_{G}$ and relate that algebra to the other algebras described in the introduction. The results are analogous to results in the theory of (nongraded) polynomial identities. We will see for example that $U_{G}$ is a prime ring and that its algebra of central quotients, the universal $G$-graded algebra, is central simple.

We start with the following lemma.

Lemma 5. Let $c$ be a nondegenerate cocycle on $G$ and let $\mathbb{C}^{c} G$ be the twisted group algebra. Let $L$ be a subfield of $\mathbb{C}$.

(a) Let $\beta: G \times G \rightarrow L^{\times}$be a two-cocycle and let $L^{\beta} G$ be the twisted group algebra. There is a homomorphism $\eta: L^{\beta} G \rightarrow \mathbb{C}^{c} G$ over $L$ of $G$-graded algebras if and only if the cocycles $c$ and $\beta$ are cohomologous over $\mathbb{C}$. In particular $\operatorname{Im}(\eta)$ is a $G$-graded form of $\mathbb{C}^{c} G$.

(b) There exists a two-cocycle $\beta: G \times G \rightarrow L^{\times}$cohomologous to $c$ over $\mathbb{C}$ if and only if $L$ contains $\mu$, the set of Proposition 1 .

Proof. (a) If $\eta: L^{\beta} G \rightarrow \mathbb{C}^{c} G$ is a homomorphism of $G$-graded $L$-algebras, then there are bases $\left\{v_{\sigma}\right\} \subset L^{\beta} G,\left\{u_{\sigma}\right\} \subset \mathbb{C}^{c} G$, and scalars $\left\{\lambda_{\sigma}\right\} \subset \mathbb{C}^{\times}$such that $v_{\sigma} v_{\tau}=\beta(\sigma, \tau) v_{\sigma \tau}, u_{\sigma} u_{\tau}=c(\sigma, \tau) u_{\sigma \tau}$ and $\eta\left(v_{\sigma}\right)=\lambda_{\sigma} u_{\sigma}$. It follows that $\beta$ and $c$ are cohomologous over $\mathbb{C}$. A similar calculation shows the other direction.

(b) We have seen in Proposition 1 that $\phi([c])=\mu$, where $\phi$ is the isomorphism between $H^{2}\left(G, \mathbb{C}^{\times}\right)$and $\operatorname{Hom}\left(M(G), \mathbb{C}^{\times}\right)$. Hence if $\beta$ is cohomologous to $c$ over $\mathbb{C}$, then $L$ must contain $\mu$ by part (d) of Proposition 1 .

For the converse we may assume that $L=\mathbb{Q}(\mu)$. By the naturality of the Universal Coefficient Theorem we have a commutative diagram

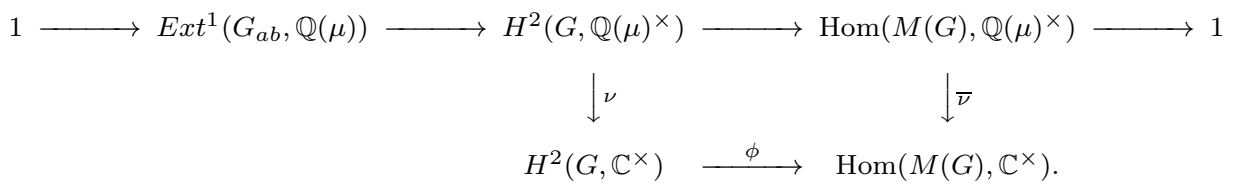

Clearly $\phi([c]) \in \operatorname{Hom}\left(M(G), \mathbb{C}^{\times}\right)$is in $\operatorname{Im}(\bar{\nu})$ and hence $[c]$ is in $\operatorname{Im}(\nu)$.

The first step in the analysis of $U_{G}$ is the construction of a counterpart to the ring of generic matrices. Let $G$ be a group of central type of order $n^{2}$ and let $c: G \times G \rightarrow \mathbb{C}^{\times}$be a nondegenerate two-cocycle. For every $g \in G$ let $t_{i g}$ for $i=1,2,3, \ldots$ be indeterminates. For each $g \in G$ let $t_{g}=t_{1 g}$. We will assume $u_{1}=1$ (i.e. the cocycle $c$ is normalized). Let $k$ denote the field generated over $\mathbb{Q}$ by the indeterminates and the values of the cocycle. Note that by Lemma 5 , $k$ contains $\mathbb{Q}(\mu)$. Consider the twisted group algebra $k^{c} G$. Because the cocycle is nondegenerate, $\mathbb{Q}(c(g, h))^{c} G$ is a central simple $\mathbb{Q}(c(g, h))$-algebra and hence $k^{c} G$ is a central simple $k$-algebra. Now let $\overline{U_{G}}$ denote the $\mathbb{Q}(\mu)$-subalgebra of $k^{c} G$ generated by the elements $t_{i g} u_{g}$ for all $i$ and all $g \in G$. We want to describe $\overline{U_{G}}$. Let $\bar{Z}$ denote the center of $\overline{U_{G}}$.

Proposition 6. (a) The center $\bar{Z}$ of $\overline{U_{G}}$ is the $\mathbb{Q}(\mu)$-subalgebra of $\overline{U_{G}}$ generated by the following set of elements:

$$
\left\{t_{i_{1} g_{1}} u_{g_{1}} t_{i_{2} g_{2}} u_{g_{2}} \cdots t_{i_{m} g_{m}} u_{g_{m}} \mid g_{1} g_{2} \cdots g_{m}=1\right\} \text {. }
$$

(b) We have $\bar{Z} \subseteq k$. In particular $\bar{Z}$ is an integral domain. 
Proof. The ring $k \overline{U_{G}}$ contains the elements $u_{g}$ for all $g \in G$ and so $k \overline{U_{G}}=k^{c} G$, which has center $k$. Hence the center of $\overline{U_{G}}$ is $\overline{U_{G}} \cap k$. But this intersection is precisely the $\mathbb{Q}(\mu)$ span of the given set of monomials. This proves both parts.

Now let $s: G \times G \rightarrow k^{\times}$be the function given by $s(g, h)=\frac{t_{g} t_{h}}{t_{g h}} c(g, h)$. This is a two-cocycle, cohomologous to $c$ over $k$. Because $c$ is nondegenerate, so is $s$, and hence the algebra $k^{s} G$ is $k$-central simple. In fact it is convenient to view the algebra $k^{s} G$ as equal to $k^{c} G$. It is spanned over $k$ by the elements $t_{g} u_{g}$.

Let $Y$ be the subgroup of $k^{\times}$generated by the values of $s$ and let $\bar{S}=\mathbb{Q}(\mu)[Y]$, a subring of $k$. The $\bar{S}$-subalgebra of $k^{s} G$ generated by the elements $t_{g} u_{g}$ is the twisted group algebra $\bar{S}^{s} G$. Let $\bar{M}=\left\{t_{g_{1}} u_{g_{1}} t_{g_{2}} u_{g_{2}} \cdots t_{g_{m}} u_{g_{m}} \mid g_{1} g_{2} \cdots g_{m}=1\right\}$. Let $\bar{F} \subseteq k$ denote the field of fractions of $\bar{Z}$.

Proposition 7. (a) The set $\bar{M}$ is a multiplicatively closed subset of $\bar{Z}$ and $\bar{M}^{-1} \bar{Z}=$ $\bar{S}\left[\frac{t_{i g}}{t_{g}} \mid i \geq 1, g \in G\right]$.

(b) We have $\overline{U_{G}}\left(\bar{M}^{-1} \bar{Z}\right)=\bar{S}^{s} G\left(\bar{M}^{-1} \bar{Z}\right)$. Moreover $\overline{U_{G} F}=\bar{S}^{s} G \bar{F}=\bar{F}^{s} G$.

(c) $\bar{F}^{s} G$ is a central simple $\bar{F}$-algebra.

(d) The ring $\overline{U_{G}}$ is a prime ring with ring of central quotients $Q\left(\overline{U_{G}}\right)$ isomorphic to the central simple algebra $\bar{F}^{s} G$.

Proof. (a) We first show $\bar{M}^{-1} \bar{Z} \supseteq \bar{S}\left[\frac{t_{i g}}{t_{g}} \mid i \geq 1, g \in G\right]$. Observe that $t_{e}$ and $t_{g} t_{g^{-1}} c\left(g, g^{-1}\right)$ are in $\bar{M}$ and hence $t_{i g} t_{g^{-1}} c\left(g, g^{-1}\right) / t_{g} t_{g^{-1}} c\left(g, g^{-1}\right)=t_{i g} / t_{g}$ is in $\bar{M}^{-1} \bar{Z}$. Next, the elements $s(g, h)^{ \pm 1}$ are in $\bar{M}^{-1} \bar{Z}$ because the elements

$$
t_{g} u_{g} t_{h} u_{h} t_{(g h)^{-1}} u_{(g h)^{-1}}=t_{g} t_{h} t_{(g h)^{-1}} c(g, h) c\left(g h,(g h)^{-1}\right)
$$

and

$$
t_{g h} u_{g h} t_{(g h)^{-1}} u_{(g h)^{-1}}=t_{g h} t_{(g h)^{-1}} c\left(g h,(g h)^{-1}\right)
$$

are in $\bar{M}$. Similar calculations show that the opposite inclusion also holds.

(b) This follows easily from part (a).

(c) We have $\bar{F}^{s} G \otimes_{\bar{F}} k=k^{s} G$, which is $k$-central simple, and so $\bar{F}^{s} G$ is $\bar{F}$-central simple.

(d) By part (b) we have $\overline{U_{G} F}=\bar{S}^{s} G \bar{F}=\bar{F}^{s} G$ and $\bar{F}^{s} G$ is $\bar{F}$-central simple, the $\operatorname{ring} \overline{U_{G}}$ is prime and because $\bar{F}$ is the ring of fractions of $\bar{Z}$ we see that the ring of central quotients $Q\left(\overline{U_{G}}\right)$ is isomorphic to $\bar{F}^{s} G$.

Let $\psi$ denote the $\mathbb{Q}(\mu)$-algebra homomorphism from the free algebra $\Sigma(\mathbb{Q}(\mu))$ to $k^{c} G$ given by $\psi\left(x_{i g}\right)=t_{i g} u_{g}$. The image of this map is clearly $\overline{U_{G}}$.

Proposition 8. The kernel of $\psi$ is the ideal $T(\mathbb{Q}(\mu))$ of graded identities of $\mathbb{C}^{c} G$ and hence $\psi$ induces a $\mathbb{Q}(\mu)$-algebra isomorphism from $U_{G}$ to $\overline{U_{G}}$. In particular the universal algebra $U_{G}$ is a prime ring with center isomorphic to $\bar{Z}$, and its ring of central quotients, that is $Q\left(U_{G}\right)$, is isomorphic to $\bar{F}^{s} G$.

Proof. This is clear since a polynomial $p\left(x_{i g}\right) \in \Sigma(\mathbb{Q}(\mu))$ is an identity of $\mathbb{C}^{c} G$ if and only if $p\left(\lambda_{i g} u_{g}\right)=0$ for any $\lambda_{i g} \in \mathbb{C}$, and this is equivalent to $p\left(t_{i g} u_{g}\right)=0$, where $\left\{t_{i g}\right\}$ are central indeterminates.

Because of this isomorphism, from now on we will drop the bars on $\bar{Z}, \bar{F}$, etc.

We want to say more about $F$, the field of fractions of the center of the universal algebra $U_{G}$. The group $Y$ is finitely generated and hence of the form $Y_{t} Y_{f}$, where 
$Y_{t}$ is the torsion subgroup of $Y$ and $Y_{f}$ is a finitely generated free abelian group. Because $Y$ is a subgroup of $k^{\times}$it follows that $Y_{t}$ is cyclic. We will see in the next proposition that in fact $Y_{t}=\mu$. Let $y_{1}, y_{2}, \ldots, y_{m} \in k$ denote a basis for $Y_{f}$. (We will see later, in Proposition 14, that the rank of $Y_{f}$ is equal to $n$, the order of $G$, and so $m=n$.)

Proposition 9. (a) We have $Y_{t}=Y \cap \mathbb{Q}(\{c(g, h) \mid g, h \in G\})=\mu$.

(b) We have $S=\mathbb{Q}(\mu)[Y]=\mathbb{Q}(\mu)\left[y_{1}^{ \pm 1}, y_{2}^{ \pm 1}, \ldots, y_{m}^{ \pm 1}\right]$, the ring of Laurent polynomials in $y_{1}, \ldots, y_{m}$ over $\mathbb{Q}(\mu)$.

(c) We have $F=\mathbb{Q}(\mu)\left(y_{1}, \ldots, y_{m}\right)\left(\frac{t_{i g}}{t_{g}} \mid i \geq 1, g \in G\right)$. In particular $F$ is isomorphic to the field of rational functions in countably many variables over $\mathbb{Q}(\mu)$.

Proof. To prove (a) we prove the following inclusions: (i) $Y_{t} \subseteq Y \cap \mathbb{Q}(\{c(g, h) \mid g, h \in$ $G\}$ ), (ii) $\mu \subseteq Y_{t}$, and (iii) $Y \cap \mathbb{Q}(\{c(g, h) \mid g, h \in G\}) \subseteq \mu$. To prove (i) consider an arbitrary element $z$ in $Y, z=\prod_{i=1}^{n} s\left(g_{i}, h_{i}\right)^{\epsilon_{i}}=\prod_{i=1}^{n}\left(\frac{t_{g_{i}} t_{h_{i}}}{t_{g_{i} h_{i}}} c\left(g_{i}, h_{i}\right)\right)^{\epsilon_{i}}$, where $\epsilon_{i}$ are \pm 1 . If the product is not independent of the $t^{\prime}$ 'say $t_{g}$ appears) it is clear that any positive power of $z$ contains a power of $t_{g}$ and hence does not equal 1 . This shows (i). Next, let $\lambda \in \mu$ and let

$$
x_{1 g_{1}} x_{1 g_{2}} \cdots x_{1 g_{k}}-\lambda x_{1 g_{\pi(1)}} x_{1 g_{\pi(2)}} \cdots x_{1 g_{\pi(k)}}
$$

be an elementary identity. It follows that

$$
t_{g_{1}} u_{g_{1}} t_{g_{2}} u_{g_{2}} \cdots t_{g_{k}} u_{g_{k}}=\lambda t_{g_{\pi(1)}} u_{g_{\pi(1)}} t_{g_{\pi(2)}} u_{g_{\pi(2)}} \cdots t_{g_{\pi(k)}} u_{g_{\pi(k)}}
$$

and hence

$$
\begin{aligned}
s\left(g_{1}, g_{2}\right) s\left(g_{1} g_{2}, g_{3}\right) & \cdots s\left(g_{1} g_{2} \cdots g_{k-1}, g_{k}\right) t_{g_{1} g_{2} \cdots g_{k}} u_{g_{1} g_{2} \cdots g_{k}} \\
& =\lambda s\left(g_{\pi(1)}, g_{\pi(2)}\right) s\left(g_{\pi(1)} g_{\pi(2)}, g_{\pi(3)}\right) \cdots \\
& s\left(g_{\pi(1)} g_{\pi(1)} \cdots g_{\pi(k-1)}, g_{\pi(k)}\right) t_{g_{\pi(1)} g_{\pi(2)} \cdots g_{\pi(k)}} u_{g_{\pi(1)} g_{\pi(2)} \cdots g_{\pi(k)}} .
\end{aligned}
$$

But $g_{1} g_{2} \cdots g_{k}=g_{\pi(1)} g_{\pi(2)} \cdots g_{\pi(k)}$ in $G$ and so $\lambda$ lies in $Y$. Because $\lambda$ is a root of unity, we get $\lambda \in Y_{t}$. This proves (ii).

To prove (iii) let $\lambda$ be an element of $Y \cap \mathbb{Q}(\{c(g, h) \mid g, h \in G\})$. We can write

$$
\begin{aligned}
\lambda & =s\left(g_{1}, h_{1}\right)^{\epsilon_{1}} s\left(g_{2}, h_{2}\right)^{\epsilon_{2}} \cdots s\left(g_{k}, h_{k}\right)^{\epsilon_{k}} \\
& =\left(\frac{t_{g_{1}} t_{h_{1}}}{t_{g_{1} h_{1}}} c\left(g_{1}, h_{1}\right)\right)^{\epsilon_{1}}\left(\frac{t_{g_{2}} t_{h_{2}}}{t_{g_{2} h_{2}}} c\left(g_{2}, h_{2}\right)\right)^{\epsilon_{2}} \cdots\left(\frac{t_{g_{k}} t_{h_{k}}}{t_{g_{k} h_{k}}} c\left(g_{k}, h_{k}\right)\right)^{\epsilon_{k}} .
\end{aligned}
$$

We use the notation and setup of part (b) of Proposition 1 Let $d$ be the element of the free group $F$ given by

$$
d=x_{g_{1}} x_{h_{1}}\left(x_{g_{1} h_{1}}\right)^{-1} x_{g_{2}} x_{h_{2}}\left(x_{g_{2} h_{2}}\right)^{-1} \cdots x_{g_{k}} x_{h_{k}}\left(x_{g_{k} h_{k}}\right)^{-1} .
$$

Clearly $d$ lies in $R$. Because $\lambda$ lies in $\mathbb{Q}(\{c(g, h) \mid g, h \in G\})$, the variables $t_{g}$ in the expression for $\lambda$ must all cancel and so $d \in[F, F]$ and $\lambda=c\left(g_{1}, h_{1}\right)^{\epsilon_{1}} c\left(g_{2}, h_{2}\right)^{\epsilon_{2}} \ldots$ $c\left(g_{k}, h_{k}\right)^{\epsilon_{k}}$. In particular we may consider the element $\bar{d}$ in $M(G)$. Then again as in part (b) of Proposition 1 we have

$$
\phi([c])(\bar{d})=\gamma(\bar{d})=c\left(g_{1}, h_{1}\right)^{\epsilon_{1}} c\left(g_{2}, h_{2}\right)^{\epsilon_{2}} \cdots c\left(g_{k}, h_{k}\right)^{\epsilon_{k}}=\lambda
$$

and so $\lambda$ lies in $\mu$.

Part (b) is clear. Part (c) follows from part (b) and from the fact that $M^{-1} Z=$ $S\left[\frac{t_{i g}}{t_{g}} \mid i \geq 1, g \in G\right]$. 
Our next goal is to study the algebra $S^{s} G$. We will see that it is Azumaya and that its simple images are the graded forms of $\mathbb{C}^{c} G=M_{n}(\mathbb{C})$.

Proposition 10. (a) Let $m$ be a maximal ideal of $S$ and let $y \rightarrow \bar{y}$ denote the canonical homomorphism from $S$ to $\tilde{S}=S / m$. Consider the two-cocycle $\tilde{s}: G \times$ $G \longrightarrow S / m$ induced by $s$ and let $\tilde{S}^{\tilde{s}} G$ be the corresponding twisted group algebra. Then the algebra $S^{s} G / m S^{s} G$ is isomorphic to $\tilde{S}^{\tilde{s}} G$ and is a central simple $\tilde{S}$ algebra.

(b) The ring $S^{s} G$ is Azumaya over $S$.

Proof. (a) We first show that the two-cocycle $\tilde{s}$ is nondegenerate. We use the following characterization of nondegeneracy (see Isaacs [15, Problem 11.8]): a twococycle $\delta: G \times G \rightarrow \mathbb{C}^{\times}$is nondegenerate if and only if for every element $g \in G$ there is an element $h \in G$ such that $g$ and $h$ commute, but in the algebra $\mathbb{C}^{\delta} G$ the elements $u_{g}$ and $u_{h}$ do not commute. Let $g \in G$. Because $c$ is nondegenerate there is an element $h \in G$ that centralizes $g$ and such that $c(g, h) c(h, g)^{-1} \neq 1$. Moreover $c(g, h) c(h, g)^{-1} \in \mu$ and $s(g, h) s(h, g)^{-1}=c(g, h) c(h, g)^{-1}$. Because $S$ contains the field $\mathbb{Q}(\mu)$, the restriction of the canonical homomorphism from $S$ to $\tilde{S}$ is injective on $\mu$ and so $\tilde{s}(g, h) \tilde{s}(h, g)^{-1} \neq 1$. Hence $\tilde{s}$ is nondegenerate. It follows that $\tilde{S}^{\tilde{s}} G$ is a central simple $\tilde{S}$-algebra. It is clear that the canonical homomorphism from $S^{s} G$ to $\tilde{S}^{\tilde{s}} G$ is surjective with kernel $m S^{s} G$.

(b) This follows from part (a) (see DeMeyer and Ingraham [10, Theorem 7.1]).

Let $L$ be any subfield of $\mathbb{C}$. Recall that every $G$-graded form of $\mathbb{C}^{c} G$ over $L$ is a twisted group algebra $L^{\beta} G$, where $\beta$ is a two-cocycle cohomologous to $c$ over $\mathbb{C}$.

We will show now that $S^{s} G$ is universal with respect to such forms. The following observation about the Hopf formula $M(G)=[F, F] \cap R /[F, R]$ will be useful (and is probably well known). We use the notation and discussion preceding Proposition 1 .

Lemma 11. Every element in $[F, F] \cap R$ is equivalent modulo $[F, R]$ to an element of the form $x_{g_{1}} \cdots x_{g_{k}}\left(x_{g_{\pi(1)}} \cdots x_{g_{\pi(k)}}\right)^{-1}$, where $\pi \in \operatorname{Sym}(k)$ and $g_{1} \cdots g_{k}=$ $g_{\pi(1)} \cdots g_{\pi(k)}$.

Proof. Let $y \in[F, F] \cap R$. We can write $y=x_{g_{1}}^{\epsilon_{1}} x_{g_{2}}^{\epsilon_{2}} \cdots x_{g_{m}}^{\epsilon_{m}}$, where $\epsilon_{i}= \pm 1$ for $1 \leq i \leq m$ and where for each group element $g$ that appears the elements $x_{g}$ and $x_{g}^{-1}$ appear the same number of times (and so $m$ is even) and where the product $g_{1}^{\epsilon_{1}} g_{2}^{\epsilon_{2}} \cdots g_{m}^{\epsilon_{m}}$ equals one. Let $m=2 r$. We claim that we may assume $\epsilon_{i}=1$ if $1 \leq i \leq r$ and $\epsilon_{i}=-1$ if $r+1 \leq i \leq m$. The lemma follows easily from this claim. To prove the claim, suppose $\epsilon_{i}=-1$ for some $i, 1 \leq i \leq r$. We consider $y=x_{g_{1}}^{\epsilon_{1}} x_{g_{2}}^{\epsilon_{2}} \cdots x_{g_{m}}^{\epsilon_{m}}=x_{g_{1}}^{\epsilon_{1}} x_{g_{2}}^{\epsilon_{2}} \cdots x_{g_{r}}^{\epsilon_{r}}\left(x_{g_{i}} x_{g_{i}^{-1}}\left(x_{g_{i}^{-1}}\right)^{-1}\left(x_{g_{i}}\right)^{-1}\right) x_{g_{r+1}}^{\epsilon_{r+1}} \cdots x_{g_{m}}^{\epsilon_{m}}$. Now for any element $g \in G$ the element $x_{g} x_{g^{-1}}$ commutes modulo $[F, R]$ with every element of $F$. Hence we can move the element $x_{g_{i}} x_{g_{i}^{-1}}$ in $y$ and obtain an element that is equivalent modulo $[F, R]$ to $y$ and for which the element $x_{g_{i}}^{-1}$ is replaced by $x_{g_{i}-1}$. Continuing in this way proves the claim.

Proposition 12. Let $L$ be a subfield of $\mathbb{C}$ which contains $\mathbb{Q}(\mu)$. Let $\varphi: S \longrightarrow L$ be a $\mathbb{Q}(\mu)$-algebra homomorphism. Let $\beta(g, h)=\varphi(s(g, h))$. Then $\varphi$ induces a $G$-graded homomorphism $\hat{\varphi}: S^{s} G \longrightarrow L^{\beta} G$, and $L^{\beta} G$ is a $G$-graded form of $\mathbb{C}^{c} G$. In particular $\beta$ is cohomologous to $c$ over $\mathbb{C}$. 
Conversely if $L^{\beta} G$ is a $G$-graded form of $\mathbb{C}^{c} G$, then there is homomorphism $\varphi$ of $S$ into $L$ such that $\gamma=\varphi(s(g, h)$ is a cocycle on $G$ cohomologous to $\beta$ over $L$ and $\varphi$ induces a homomorphism from $S^{s} G$ onto $\varphi(S)^{\gamma} G$, a G-graded form of $L^{\beta} G$.

Proof. Let $\varphi: S \longrightarrow L$ be a $\mathbb{Q}(\mu)$-algebra homomorphism and let $\beta(g, h)=$ $\varphi(s(g, h))$. We have seen in Proposition 10 that $\beta$ is a nondegenerate cocycle on $G$ and hence that $L^{\beta} G$ is a central simple $L$-algebra. Clearly $\varphi$ induces a homomorphism $\hat{\varphi}: S^{s} G \longrightarrow L^{\beta} G$, and if $A$ denotes the image of $S^{s} G$ and $A$ has center $R$ (say), then $A \otimes_{R} L=L^{\beta} G$. We are left with showing $L^{\beta} G$ is a form of $\mathbb{C}^{c} G$; that is, we need to show $\beta$ and $c$ are cohomologous over $\mathbb{C}$. It suffices to show that they determine the same element in $\operatorname{Hom}\left(M(G), \mathbb{C}^{\times}\right)$, that is, that $\phi([c])=\phi([\beta])$, where $\phi$ is the isomorphism from $H^{2}\left(G, \mathbb{C}^{\times}\right)$to $\operatorname{Hom}\left(M(G), \mathbb{C}^{\times}\right)$(see the discussion preceding Proposition 11). By the lemma it suffices to show that if $g_{1} \cdots g_{k}=g_{\pi(1)} \cdots g_{\pi(k)}$, then $\phi([\beta])(y)=\phi([c])(y)$, where $y=\left(x_{g_{1}} \cdots x_{g_{k}}\right)\left(x_{g_{\pi(1)}} \cdots x_{g_{\pi(k)}}\right)^{-1}$. For the purposes of the proof it will be useful to use the following notation: If $\alpha$ is any twococycle, then we denote the expression

$$
\frac{\alpha\left(g_{1}, g_{2}\right) \alpha\left(g_{1} g_{2}, g_{3}\right) \cdots \alpha\left(g_{1} g_{2} \ldots g_{k-1}, g_{k}\right)}{\alpha\left(g_{\pi(1)}, g_{\pi(2)}\right) \alpha\left(g_{\pi(1)} g_{\pi(2)}, g_{\pi(3)}\right) \cdots \alpha\left(g_{\pi(1)} g_{\pi(2)} \cdots g_{\pi(k-1)}, g_{\pi(k)}\right)}
$$

by $\alpha\left(g_{1}, g_{2}, \ldots, g_{k}\right) / \alpha\left(g_{\pi(1)}, g_{\pi(2)}, \ldots, g_{\pi(k)}\right)$.

We have

$$
\begin{aligned}
\phi([\beta])(y)= & \beta\left(g_{1}, g_{2}, \ldots, g_{k}\right) / \beta\left(g_{\pi(1)}, g_{\pi(2)}, \ldots, g_{\pi(k)}\right) \\
= & \varphi\left(s\left(g_{1}, g_{2}, \ldots, g_{k}\right) / s\left(g_{\pi(1)}, g_{\pi(2)}, \ldots, g_{\pi(k)}\right)\right) \\
= & \varphi\left(\left(t_{g_{1}} t_{g_{2}} / t_{g_{1} g_{2}}\right)\left(t_{g_{1} g_{2}} t_{g_{3}} / t_{g_{1} g_{2} g_{3}}\right) \cdots\left(t_{g_{1} g_{2} \cdots g_{k-1}} t_{g_{k}} / t_{g_{1} g_{2} \cdots g_{k}}\right) /\right. \\
& \left(t_{g_{\pi(1)}} t_{g_{\pi(2)}} / t_{g_{\pi(1)}} g_{\pi(2)}\right) \\
& \cdots\left(t_{g_{\pi(1)} g_{\pi(2)} \cdots g_{\pi(k-1)}} t_{g_{\pi(k)}} / t_{\left.g_{\pi(1)} g_{\pi(2)} \cdots g_{\pi(k)}\right)}\right) \\
& \left.c\left(g_{1}, g_{2}, \ldots, g_{k}\right) / c\left(g_{\pi(1)}, g_{\pi(2)}, \ldots, g_{\pi(k)}\right)\right) \\
= & \varphi\left(\left(t_{g_{1} g_{2} \cdots g_{k}} / t_{g_{\pi(1)}} g_{\pi(2)} \cdots g_{\pi(k)}\right) c\left(g_{1}, g_{2}, \ldots, g_{k}\right) / c\left(g_{\pi(1)}, g_{\pi(2)}, \ldots, g_{\pi(k)}\right)\right) \\
= & \varphi\left(c\left(g_{1}, g_{2}, \ldots, g_{k}\right) / c\left(g_{\pi(1)}, g_{\pi(2)}, \ldots, g_{\pi(k)}\right)\right),
\end{aligned}
$$

because $g_{1} \cdots g_{k}=g_{\pi(1)} \cdots g_{\pi(k)}$. But this last expression is exactly $\phi([c])(y)$, as desired.

For the converse assume $L^{\beta} G$ is a $G$-graded form of $\mathbb{C}^{c} G$. Then $L^{\beta} G$ satisfies the graded identities of $M_{n}(\mathbb{C})$, and so there is a graded homomorphism from $U_{G}$ sending each $x_{i g}$ to $u_{g}$ in $L^{\beta} G$. But under this map the images of the elements of $M$ are nonzero elements in $L$ and so we get an induced homomorphism from $S^{s} G$ that takes $S$ into $L$. The result follows.

We can use this result to obtain a parametrization of the $G$-graded forms over $L$. We begin with a homomorphism $\varphi$ from $S$ to $L$ and the two-cocycle $\beta$ given by $\beta(g, h)=\varphi(s(g, h))$. In the previous proposition we say that $\beta$ is cohomologous to $c$ over $\mathbb{C}$ and so $L^{\beta} G$ is a form of $M_{n}(\mathbb{C})$. We will now show how to produce all other forms over $L$. Let $V$ be the free abelian group (of rank ord $(G)$ ) on symbols $r_{g}, g \in G$. Let $U$ be the subgroup generated by the elements $r_{g} r_{h} / r_{g h}$. Note that $V / U$ is a finite group, isomorphic to $G / G^{\prime}=G_{a b}$ via the map that sends $r_{g}$ to $g G^{\prime}$. For each element $\psi$ in $\operatorname{Hom}\left(U, L^{\times}\right)$, let $\beta_{\psi}$ be given by $\beta_{\psi}(g, h)=\beta(g, h) \psi\left(r_{g} r_{h} / r_{g h}\right)$. Note that there is a canonical homomorphism from $\operatorname{Hom}\left(V, L^{\times}\right)$to $\operatorname{Hom}\left(U, L^{\times}\right)$. We will let $\operatorname{Im}\left(\left(\operatorname{Hom}\left(V, L^{\times}\right)\right)\right.$denote its image in $\operatorname{Hom}\left(U, L^{\times}\right)$. 
Proposition 13. The following hold:

(a) For every $\psi \in \operatorname{Hom}\left(U, L^{\times}\right)$the function $\beta_{\psi}$ is a two-cocycle cohomologous to $\beta$ over $\mathbb{C}$. In particular $L^{\beta_{\psi}} G$ is a $G$-graded form of $M_{n}(\mathbb{C})$.

(b) If $L^{\gamma} G$ is a $G$-graded form of $M_{n}(\mathbb{C})$ there is a homomorphism $\psi \in$ $\operatorname{Hom}\left(U, L^{\times}\right)$such that $\gamma=\beta_{\psi}$. In particular $L^{\gamma} G$ is isomorphic to $L^{\beta_{\psi}} G$ as a $G$-graded algebra.

(c) Two forms $L^{\beta_{\psi}} G$ and $L^{\beta_{\psi^{\prime}}} G$ are $G$-graded isomorphic if and only if $\beta_{\psi}=$ $f(\alpha) \beta_{\psi^{\prime}}$ for some $\alpha$ in $\operatorname{Im}\left(\left(\operatorname{Hom}\left(V, L^{\times}\right)\right)\right.$.

(d) The function $\psi \rightarrow \beta_{\psi}$ induces a one-to-one correspondence between the group $\operatorname{Hom}\left(U, L^{\times}\right) / \operatorname{Im}\left(\operatorname{Hom}\left(V, L^{\times}\right)\right.$and the set of $G$-graded isomorphism classes of $G$ graded forms of $\mathbb{C}^{c} G$ over $L$.

Proof. (a) It is clear that $\beta_{\psi}$ is a cocycle. To show that $\beta$ and $\beta_{\psi}$ are cohomologous over $\mathbb{C}$ it suffices to show that they determine the same element in $\operatorname{Hom}\left(M(G), \mathbb{C}^{\times}\right)$, that is, that $\phi\left(\left[\beta_{\psi}\right]\right)=\phi([\beta])$, where $\phi$ is the isomorphism from $H^{2}\left(G, \mathbb{C}^{\times}\right)$to $\operatorname{Hom}\left(M(G), \mathbb{C}^{\times}\right)$. The argument is quite similar to the proof of the corresponding fact in Proposition 12, so we will omit it.

(b) If $L^{\gamma} G$ is a $G$-graded form of $M_{n}(\mathbb{C})$, then we have seen that $\gamma$ is cohomologous to $\beta$ over $\mathbb{C}$, so there is a cochain $\lambda: G \rightarrow \mathbb{C}^{\times}$such that for all $g, h \in G$, we have $\gamma(g, h)=(\lambda(g) \lambda(h) / \lambda(g h)) \beta(g, h)$. It follows that for all $g, h \in G, \lambda(g) \lambda(h) / \lambda(g h)$ is in $L$. The cochain $\lambda$ clearly determines a homomorphism from $V$ to $\mathbb{C}^{\times}$(sending $r_{g}$ to $\left.\lambda(g)\right)$ and this homomorphism restricts to a homomorphism $\psi$ from $U$ to $L^{\times}$ (given by $\psi\left(r_{g} r_{h} / r_{g h}\right)=\lambda(g) \lambda(h) / \lambda(g h)$ ) which satisfies $\gamma=\beta_{\psi}$.

(c) If $L^{\beta_{\psi}} G$ and $L^{\beta_{\psi^{\prime}}} G$ are $G$-graded isomorphic, then $\beta_{\psi}$ is cohomologous to $\beta_{\psi^{\prime}}$ over $L$. As in the proof of part (b) this means there is a homomorphism $\alpha$ from $V$ to $L^{\times}$such that $\beta_{\psi}(g, h)=(\alpha(g) \alpha(h) / \alpha(g h)) \beta_{\psi^{\prime}}$. It follows that $\psi=\alpha \psi^{\prime}$, as desired.

(d) This follows from the first three parts.

We can connect this parametrization with cohomology as follows. We have a short exact sequence of groups

$$
1 \longrightarrow U \longrightarrow V \longrightarrow G_{a b} \longrightarrow 1
$$

Applying the functor $\operatorname{Hom}\left(-, L^{\times}\right)$we obtain a long exact sequence in cohomology

$$
\cdots \longrightarrow \operatorname{Hom}\left(V, L^{\times}\right) \longrightarrow \operatorname{Hom}\left(U, L^{\times}\right) \longrightarrow \operatorname{Ext}\left(G_{a b}, L^{\times}\right) \longrightarrow 1,
$$

where the surjectivity of the last map follows from the fact that $\operatorname{Ext}\left(V, L^{\times}\right)=0$ because $V$ is a free abelian group. We also have the universal coefficient sequence:

$$
1 \rightarrow \operatorname{Ext}^{1}\left(G_{a b}, L^{\times}\right) \rightarrow H^{2}\left(G, L^{\times}\right) \rightarrow \operatorname{Hom}\left(M(G), L^{\times}\right) \rightarrow 1 .
$$

Combining these equations shows that $\operatorname{Hom}\left(U, L^{\times}\right) / \operatorname{Im}\left(\operatorname{Hom}\left(V, L^{\times}\right)\right)$is isomorphic to $\operatorname{Ext}^{1}\left(G_{a b}, L^{\times}\right)$, the kernel of the map from $H^{2}\left(G, L^{\times}\right)$to $\operatorname{Hom}\left(M(G), L^{\times}\right)$.

The last result in this section is the determination of the rank of $Y_{f}$, the free part of the subgroup of $k^{\times}$generated by the values of $s$. The computation involves the groups $U$ and $V$. We first remark that because $V / U$ is finite, the rank of $U$ is equal to the rank of $V$, which is clearly $n$, the order of $G$.

Proposition 14. The group $Y_{f}$ is isomorphic to $U$. In particular the rank of $Y_{f}$ is $n$, the order of $G$. 
Proof. Recall that $Y$ denotes the subgroup of $k^{\times}$generated by the values of the cocycle $s$. Let $H$ denote the subgroup of $k^{\times}$generated by the values of the cocycle c. Then by Proposition 9, $Y \cap H=Y \cap \mathbb{C}^{\times}=\mu$. Let $\tilde{U}$ be the subgroup of $k^{\times}$ generated by the set $\left\{t_{g} t_{h} / t_{g h} \mid g, h \in G\right\}$. Clearly the group $\tilde{U}$ is isomorphic to $U$. Now we have $Y_{f} \cong Y / Y_{t} \cong Y / Y \cap H \cong Y H / H$. But $Y H=\tilde{U} H$. Hence $Y H / H=\tilde{U} H / H \cong \tilde{U} / \tilde{U} \cap H \cong \tilde{U}$ because $\tilde{U} \cap H=1$. Hence $Y_{f} \cong \tilde{U} \cong U$, as desired.

Corollary 15. The field of fractions of $S$ has transcendence degree $n$ over $\mathbb{Q}(\mu)$.

Proof. This follows from the description of $S$ given in Proposition 9

\section{The universal $G$-Graded Central simple algebra}

We have seen that each fine grading by a group $G$ on $M_{n}(\mathbb{C})$ gives rise to a nondegenerate two-cocycle $c$ on the group $G$ (which must be of central type) such that $M_{n}(\mathbb{C})$ is graded isomorphic to $\mathbb{C}^{c} G$. We have also seen that the universal $G$-graded algebra $U_{G}$ is a prime ring with ring of central quotients $Q\left(U_{G}\right)$ graded isomorphic to the $F$-central simple algebra $F^{s} G$, where $s$ is the generic cocycle obtained from $c$ and $F=\mathbb{Q}(\mu)\left(y_{1}, \ldots, y_{m}\right)\left(\frac{t_{i g}}{t_{g}} \mid i \geq 1, g \in G\right)$ (see Proposition 9). In this section we obtain information about the index of $Q\left(U_{G}\right)$ and about the dependence of $Q\left(U_{G}\right)$ on the given cocycle $c$.

Proposition 16. The index of $Q\left(U_{G}\right)$ is equal to the maximum of the indices of the $G$-graded forms $L^{\beta} G$, where $L$ varies over all subfields of $\mathbb{C}$ that contain $\mathbb{Q}(\mu)$ and $\beta$ varies over all nondegenerate cocycles over $L$ cohomologous to $c$ over $\mathbb{C}$.

Proof. Let $M$ denote the maximum of the indices of the forms. We first claim that $\operatorname{ind}\left(Q\left(U_{G}\right)\right) \leq M$. In fact we can specialize the universal algebra $U_{G}$ by sending $x_{g}$ to $r_{g} u_{g}$, where the elements $\left\{r_{g} \mid g \in G\right\}$ are algebraically independent complex numbers. The resulting algebra $\bar{U}$ is isomorphic to $U_{G}$ and tensoring with the field of fractions of its center gives a central simple algebra that is isomorphic to $Q\left(U_{G}\right)$. Clearly the index of this form equals the index of $Q\left(U_{G}\right)$ and so we get the inequality. We proceed to show that the index of $Q\left(U_{G}\right)=F^{s} G$ is greater than or equal to $M$. Recall (Proposition 9) that the center $S$ of $S^{s} G$ is a ring of Laurent polynomials over the field $\mathbb{Q}(\mu)$. If $M$ is any maximal ideal of $S$, then $S_{M}$ is a regular local ring. By Proposition 12 it suffices to show that if $M$ is any maximal ideal of $S$, then the index of the algebra $S^{s} G \otimes_{S} F=F^{s} G$ is greater than or equal to the index of the residue algebra $S^{s} G / M S^{s} G$. This is a well-known fact. We outline a proof: Let $r_{1}, r_{2}, \ldots, r_{m}$ be a system of parameters of the regular local ring $R=S_{M}$ and let $F^{s} G=M_{t}(D)$, where $D$ is an $F$-central division algebra (so $\left.\operatorname{ind}\left(F^{s} G\right)=\operatorname{ord}(G)^{1 / 2} / t\right)$. The localization $R_{\left(r_{1}\right)}$ is a discrete valuation ring with residue field the field of fractions of the regular local ring $R_{1}=R /\left(r_{1}\right)$. The ring $R_{\left(r_{1}\right)}^{s} G$ is an Azumaya algebra over the discrete valuation $\operatorname{ring} R_{\left(r_{1}\right)}$ and hence is isomorphic to $M_{t}(A)$, where $A$ is an Azumaya algebra such that $A \otimes F=D$ (see Reiner [18, Theorem 21.6]). It follows that $R_{1}^{s} G$ is an Azumaya algebra, and if we let $Q\left(R_{1}\right)$ denote the field of fractions of $R_{1}$, then $R_{1}^{s} G \otimes Q\left(R_{1}\right)$ is isomorphic to $M_{t}\left(D_{1}\right)$, where $D_{1}$ is a central simple $Q\left(R_{1}\right)$-algebra, the residue algebra of $D$. The image of $M$ in $R_{1}$ is generated by the images of $r_{2}, r_{3}, \ldots, r_{m}$ and these $m-1$ elements form a system of parameters for $R_{1}$. We can therefore repeat the process and eventually obtain that $S_{M}^{s} G / M S_{M}^{s} G$ is isomorphic to an algebra of the form 
$M_{t}(E)$ for some central simple $S / M$-algebra $E$. Hence the index of $S_{M}^{s} G / M S_{M}^{s} G$ is at most the index of $F^{s} G$.

The question for which groups $G$ of central type there is a cocycle $c$ such that $Q\left(U_{G, c}\right)$ is a division algebra was answered in Aljadeff, et al. 2] and Natapov [17]. We consider the following list of $p$-groups, called $\Lambda_{p}$ :

(1) $G$ is abelian of symmetric type, that is, $G \cong \prod\left(C_{p^{n_{i}}} \times C_{p^{n_{i}}}\right)$,

(2) $G \cong G_{1} \times G_{2}$, where

$G_{1}=C_{p^{n}} \rtimes C_{p^{n}}=\langle\pi, \sigma| \sigma^{p^{n}}=\pi^{p^{n}}=1$ and $\left.\sigma \pi \sigma^{-1}=\pi^{p^{s}+1}\right\rangle$, where $1 \leq s<n$ and $1 \neq s$ if $p=2$, and

$G_{2}$ is an abelian group of symmetric type of exponent $\leq p^{s}$,

(3) $G \cong G_{1} \times G_{2}$, where

$G_{1}=C_{2^{n+1}} \rtimes\left(C_{2^{n}} \times Z_{2}\right)=\left\langle\begin{array}{l|l}\pi, \sigma, \tau & \begin{array}{l}\pi^{2^{n+1}}=\sigma^{2^{n}}=\tau^{2}=1, \sigma \tau=\tau \sigma, \\ \sigma \pi \sigma^{-1}=\pi^{3}, \tau \pi \tau^{-1}=\pi^{-1}\end{array}\end{array}\right\rangle$

and

$G_{2}$ is an abelian group of symmetric type of exponent $\leq 2$.

We let $\Lambda$ be the collection of nilpotent groups such that for any prime $p$, the Sylow $p$-subgroup is on the $\Lambda_{p}$.

Proposition 17. Let $G$ be a group of central type of order $n^{2}$. The following conditions are equivalent:

(a) There is a nondegenerate cocycle $c$ on $G$ for which the universal $G$-graded algebra $U_{G, c}$ is a domain.

(b) There is a nondegenerate cocycle $c$ on $G$ for which the universal central simple algebra $Q\left(U_{G, c}\right)$ is a division algebra.

(c) There is a nondegenerate cocycle c on $G$ such that the resulting $G$-graded algebra $M_{n}(\mathbb{C})$ has a $G$-graded form that is a division algebra.

(d) The group $G$ is on the list $\Lambda$.

Proof. The equivalence of (a) and (b) follows from the fact that $F^{s} G$ is isomorphic to $Q\left(U_{G, c}\right)$. The equivalence of (b) and (c) follows from the previous proposition. Finally the equivalence of (c) and (d) follows from Aljadeff et al. [2, Corollary 3] and Natapov [17, Theorem 3].

Our main result in this section is that if $G$ is a group on the list $\Lambda$, then the universal central simple $G$-graded algebra $Q\left(U_{G, c}\right)$ is independent (up to a nongraded isomorphism) of the cocycle $c$. In fact we will show that for groups $G$ on the list the automorphism group of $G$ acts transitively on the set of classes of nondegenerate cocycles.

To begin let $G$ be any central type group (not necessarily on $\Lambda$ ) and let $c$ be a nondegenerate two-cocycle on $G$ with coefficients in $\mathbb{C}^{\times}$. Let $\varphi$ be an automorphism of $G$ and let $\varphi(c)$ be the two-cocycle defined by $\varphi(c)(\sigma, \tau)=c\left(\varphi^{-1}(\sigma), \varphi^{-1}(\tau)\right)$. It is clear that $\varphi(c)$ is also nondegenerate.

Theorem 18. If $G$ is a group on the list $\Lambda$ and $c$ and $c^{\prime}$ are nondegenerate cocycles on $G$ with values in $\mathbb{C}^{\times}$, then there is an automorphism $\varphi$ of $G$ such that $\varphi(c)$ is cohomologous over $\mathbb{C}$ to $c^{\prime}$.

Proof. Let $G$ be a group on the list $\Lambda$. Because $G$ is necessarily nilpotent, we can assume that $G$ lies on $\Lambda_{p}$ for some prime $p$. In the course of the proof, whenever 
we refer to basis elements $\left\{u_{g}\right\}_{g \in G}$ in the twisted group algebra $\mathbb{C}^{c} G$, we assume they satisfy $u_{g} u_{h}=c(g, h) u_{g h}$.

The strategy is as follows: For each group $G \in \Lambda_{p}$ we fix a set of generators $\Phi$. Then we consider the family of sets of generators $\Phi^{\prime}=\varphi(\Phi)$ that are obtained from $\Phi$ via an automorphism $\varphi$ of $G$. We say that $\Phi$ and $\Phi^{\prime}$ are equivalent. Next, we exhibit a certain nondegenerate cohomology class $\alpha \in H^{2}\left(G, \mathbb{C}^{\times}\right)$by setting a set of equations (denoted by $E_{G}$ ) satisfied by elements $\left\{u_{g}\right\}_{g \in \Phi}$ in $\mathbb{C}^{c_{0}} G$, where $c_{0}$ is a two-cocycle representing $\alpha$. We say that the cohomology class $\alpha$ (or by abuse of language, the two-cocycle $c_{0}$ ) is of "standard form" with respect to $\Phi$. It is indeed abuse of language since in general the equations do not determine the two-cocycle $c_{0}$ uniquely but only its cohomology class. Finally we show that for any nondegenerate two-cocycle $c$ with values in $\mathbb{C}^{\times}$there is a set of generators $\Phi^{\prime}=\varphi(\Phi)$ with respect to which $c$ is of standard form. The desired automorphism of the group $G$ will be determined by compositions of "elementary" automorphisms, that is, automorphisms that are defined by replacing some elements of the generating set. We start with $\Phi^{(0)}=\Phi$ and denote the updated generating sets by $\Phi^{(r)}=\left\{g_{1}^{(r)}, \ldots, g_{n}^{(r)}\right\}, r=1,2,3, \ldots$ Elements in the generating set that are not mentioned remain unchanged, that is, $g_{i}^{(r)}=g_{i}^{(r-1)}$. In all steps it will be an easy check that the map defined is indeed an automorphism of $G$.

(I) We start with abelian groups on $\Lambda_{p}$. Let $G=C_{p^{r_{1}}} \times C_{p^{r_{1}}} \times C_{p^{r_{2}}} \times C_{p^{r_{2}}} \times$ $\cdots \times C_{p^{r m}} \times C_{p^{r_{m}}}$ and let $\Phi=\left\{\gamma_{1}, \gamma_{2}, \ldots, \gamma_{2 m}\right\}$ be an ordered set of generators.

Fixing a primitive $p^{n}$-th root of unity $\varepsilon$, where $n \geq \max \left(r_{k}\right), 1 \leq k \leq m$, we say that a two-cocycle $c_{0}$ is of standard form with respect to $\Phi$ if there are elements $\left\{u_{g}\right\}_{g \in \Phi}$ in $\mathbb{C}^{c_{0}} G$ that satisfy

$$
\left\{\begin{array}{l}
\left(u_{\gamma_{2 k-1}}, u_{\gamma_{2 k}}\right)=\varepsilon^{p^{n-r_{k}}} \text { for all } 1 \leq k \leq m, \\
\text { all other commutators of the } u_{\gamma_{i}} \text { 's are trivial. }
\end{array}\right.
$$

Note that $\left[c_{0}\right]$ is determined by (1). Given any nondegenerate two-cocycle $c$ it is known that there is a set of generators $\Phi^{\prime}=\varphi(\Phi)$ with respect to which $c$ is of standard form (see, for example, Aljadeff and Sonn [6. Theorem 1.1]). Hence we are done in this case.

(II) Next we consider the group $G=C_{p^{n}} \rtimes C_{p^{n}} \times C_{p^{r_{1}}} \times C_{p^{r_{1}}} \times \cdots \times C_{p^{r_{m}}} \times C_{p^{r_{m}}}$ with a set of generators $\Phi=\left\{\pi, \sigma, \gamma_{1}, \ldots, \gamma_{2 m}\right\}$, where $\pi, \sigma$ satisfy $\sigma(\pi)=\pi^{p^{s}+1}$ for some $s=1, \ldots, n-1$ if $p$ is odd and for some $s=2, \ldots, n-1$ if $p=2$, and $r_{k} \leq s$ for all $1 \leq k \leq m$. We write $G=G_{1} \times G_{2}$, where $G_{1}=C_{p^{n}} \rtimes C_{p^{n}}$.

Fix a primitive $p^{n}$-th root of unity $\varepsilon$. There exist a two-cocycle $c_{1} \in Z^{2}\left(G_{1}, \mathbb{C}^{\times}\right)$ and elements $\left\{u_{g}\right\}_{g \in\{\pi, \sigma\}}$ in $\mathbb{C}^{c_{1}} G_{1}$ that satisfy

$$
u_{\pi}^{p^{n}}=u_{\sigma}^{p^{n}}=1, u_{\sigma} u_{\pi} u_{\sigma}^{-1}=\varepsilon u_{\pi}^{p^{s+1}} .
$$

Moreover the class $\left[c_{1}\right] \in H^{2}\left(G_{1}, \mathbb{C}^{\times}\right)$is uniquely determined by (2) (see, for example, the proof of Karpilovsky [16, Theorem 10.1.25]).

Now, by Karpilovsky ([16, Proposition 10.6.1]) we have $M(G) \cong M\left(G_{1}\right) \times$ $M\left(G_{2}\right) \times\left(G_{1}^{a b} \otimes G_{2}^{a b}\right)$ (here $G^{a b}$ denotes the abelianization of $G$ ) and hence there exist a two-cocycle $c_{0} \in Z^{2}\left(G, \mathbb{C}^{\times}\right)$and elements $\left\{u_{g}\right\}_{g \in \Phi}$ in $\mathbb{C}^{c_{0}} G$ that satisfy equations (21), (11) and $u_{\pi} u_{\gamma_{i}}=u_{\gamma_{i}} u_{\pi}, u_{\sigma} u_{\gamma_{i}}=u_{\gamma_{i}} u_{\sigma}$. We denote this set of equations by $E_{G}$. The class $\left[c_{0}\right] \in H^{2}\left(G, \mathbb{C}^{\times}\right)$is uniquely determined by $E_{G}$ and is 
easily seen to be nondegenerate. We say that a class $\left[c_{0}\right]$ is of standard form with respect to $\Phi$ if there are elements $\left\{u_{g}\right\}_{g \in \Phi}$ in $\mathbb{C}^{c_{0}} G$ that satisfy $E_{G}$.

Now, let $c \in Z^{2}\left(G, \mathbb{C}^{\times}\right)$be any nondegenerate two-cocycle, and let $u_{g}$ be representatives of elements of $G$ in $\mathbb{C}^{c} G$. As explained above we set $\Phi^{(0)}=\left\{\pi^{(0)}, \sigma^{(0)}\right.$, $\left.\gamma_{1}^{(0)}, \ldots, \gamma_{2 m}^{(0)}\right\}=\Phi=\left\{\pi, \sigma, \gamma_{1}, \ldots, \gamma_{2 m}\right\}$. We are to exhibit a sequence of automorphisms such that their composition applied to $\Phi^{(0)}$ yields a generating set with respect to which the cocycle $c$ is of standard form. First, note that we may assume (by passing to an equivalent cocycle, if necessary) that $u_{\pi^{(0)}}^{p^{n}}=u_{\sigma^{(0)}}^{p^{n}}=1$. Let $\alpha \in \mathbb{C}^{\times}$ be determined by $u_{\sigma^{(0)}} u_{\pi^{(0)}} u_{\sigma^{(0)}}^{-1}=\alpha u_{\pi^{(0)}}^{p^{s}+1}$. It follows that $u_{\sigma^{(0)}} u_{\pi^{(0)}}^{p^{n-1}} u_{\sigma^{(0)}}^{-1}=$ $\alpha^{p^{n-1}} u_{\pi^{(0)}}^{p^{n-1}}$ and hence, $\alpha^{p^{n-1}}$ is a $p$-th root of unity. Next, observe that since $c$ is nondegenerate, $u_{\sigma^{(0)}}$ cannot commute with $u_{\pi^{(0)}}^{p^{n-1}}$ (for otherwise, $u_{\pi^{(0)}}^{p^{n-1}}$ is contained in the center of the algebra) and hence $\alpha$ is a primitive $p^{n}$-th root of unity. Now, replacing $\pi^{(0)}$ by a suitable prime to $p$ power of $\pi^{(0)}$ (that is, $\pi^{(1)}=\left(\pi^{(0)}\right)^{l}$, $l$ prime to $p$ ), we may assume that $\alpha=\varepsilon$. Leaving $\sigma^{(0)}$ and all other generators unchanged, that is, $\sigma^{(1)}=\sigma^{(0)}, \gamma_{1}^{(1)}=\gamma_{1}^{(0)}, \ldots, \gamma_{2 m}^{(1)}=\gamma_{2 m}^{(0)}$, we obtain a generating set $\Phi^{(1)}=\left\{\pi^{(1)}, \sigma^{(1)}, \gamma_{1}^{(1)}, \ldots, \gamma_{2 m}^{(1)}\right\}$ of $G$, equivalent to $\Phi$, such that the elements $u_{\pi^{(1)}}, u_{\sigma^{(1)}}$ in $\mathbb{C}^{c} G_{1}$ satisfy (2).

Assume $\left(u_{\gamma_{i}^{(1)}}, u_{\pi^{(1)}}\right)=\xi_{i} \neq 1$ (clearly $\xi_{i}$ is a root of unity of order $p^{r}(\leq$ $\left.\left.\operatorname{ord}\left(\gamma_{i}^{(1)}\right) \leq p^{s}\right)\right)$. Now, by the nondegeneracy of $c$, the root of unity $\zeta$ determined by the equation $u_{\sigma^{(1)}}^{p^{n-s}} u_{\pi^{(1)}} u_{\sigma^{(1)}}^{-p^{n-s}}=\zeta u_{\pi^{(1)}}$ is of order $p^{s}$ and hence $\xi_{i}=\zeta^{l}$ for some $l$. Observe that $\operatorname{ord}\left(\left(\left(\sigma^{(1)}\right)^{p^{n-s}}\right)^{l}\right) \leq p^{r}$, and hence we may put $\gamma_{i}^{(2)}=\gamma_{i}^{(1)}\left(\sigma^{(1)}\right)^{-l p^{n-s}}$ and get $\left(u_{\gamma_{i}^{(2)}}, u_{\pi^{(1)}}\right)=1$. Performing this process for all $1 \leq i \leq 2 m$ (and leaving $\pi^{(1)}$ and $\sigma^{(1)}$ unchanged) we get a set of generators $\Phi^{(2)}$ such that $\left(u_{\gamma_{i}^{(2)}}, u_{\pi^{(2)}}\right)=1$ for all $i$.

Next, if $\left(u_{\gamma_{i}^{(2)}}, u_{\sigma^{(2)}}\right) \neq 1$ we set $\gamma_{i}^{(3)}=\gamma_{i}^{(2)}\left(\pi^{(2)}\right)^{-t p^{n-s}}$ for an appropriate positive integer $t$ and get $\left(u_{\gamma_{i}^{(3)}}, u_{\sigma^{(2)}}\right)=1$. Performing this process for all $1 \leq i \leq 2 m$ we get a set of generators $\Phi^{(3)}$ such that $\left(u_{\gamma_{i}^{(3)}}, u_{\sigma^{(3)}}\right)=1$ for all $i$.

Finally, we may proceed as in Case I and obtain a generating set $\Phi^{(4)}$, equivalent to $\Phi$, with respect to which $c$ is of standard form.

(III) Next we consider the group $G=C_{2^{n+1}} \rtimes\left(C_{2^{n}} \times C_{2}\right) \times C_{2} \times C_{2} \ldots \times C_{2} \times C_{2}$, $n \geq 2$, with a set of generators $\Phi=\left\{\pi, \sigma, \tau, \gamma_{1}, \ldots, \gamma_{2 m}\right\}$, where $\sigma \pi \sigma^{-1}=\pi^{3}$ and $\tau \pi \tau^{-1}=\pi^{-1}$. We write $G=G_{1} \times G_{2}$, where $G_{1}=C_{2^{n+1}} \rtimes\left(C_{2^{n}} \times C_{2}\right)$ and $G_{2}$ is elementary abelian of rank $2 m$.

We first exhibit a construction of a field $K$ and a cocycle $\beta \in Z^{2}\left(G_{1}, K^{\times}\right)$ such that the algebra $D \cong K^{\beta} G_{1}$ is $K$-central simple (in fact a $K$-central division algebra), which is analogous to that in Natapov [17. Let $K=\mathbb{Q}(s, t)$ be the subfield of $\mathbb{C}$ generated by algebraically independent elements $s$ and $t$. Let $L=K\left(v_{\pi}\right) / K$ be a cyclotomic extension, where $v_{\pi}^{2^{n+1}}=-1$. The Galois action of $\operatorname{Gal}(L / K) \cong$ $Z_{2^{n}} \times Z_{2}=\langle\sigma, \tau\rangle$ on $L$ is given by

$$
\sigma\left(v_{\pi}\right)=v_{\pi}^{3} \text { and } \tau\left(v_{\pi}\right)=v_{\pi}^{-1} .
$$

The algebra $D$ is the crossed product $D=(L / K,\langle\sigma, \tau\rangle)$ determined by the following relations:

$$
v_{\sigma}^{2^{n}}=s, v_{\tau}^{2}=t,\left(v_{\sigma}, v_{\tau}\right)=1,
$$


where $v_{\sigma}$ and $v_{\tau}$ represent $\sigma$ and $\tau$ in $D$. It is easy to see that $D$ is isomorphic to a twisted group algebra of the form $K^{\beta} G_{1}$.

Fix a primitive $2^{n+1}$-th root of unity $\varepsilon$, and let $\xi$ be a square root of $\varepsilon$. Let $\widetilde{s}$ and $\widetilde{t}$ be elements of $\mathbb{C}$ such that $\widetilde{s}^{2^{n}}=s^{-1}$ and $\widetilde{t}^{2}=t^{-1}$. Then the elements $u_{\pi^{i} \sigma^{j} \tau^{k}}=\left(\xi v_{\pi}\right)^{i}\left(\widetilde{s} v_{\sigma}\right)^{j}\left(\widetilde{t} v_{\tau}\right)^{k}$ in $\mathbb{C}^{\beta} G_{1} \cong \mathbb{C} \otimes_{K} D$ satisfy

$$
u_{\pi}^{2^{n+1}}=u_{\sigma}^{2^{n}}=u_{\tau}^{2}=1, u_{\sigma} u_{\pi}=\varepsilon u_{\pi}^{3} u_{\sigma}, u_{\tau} u_{\pi}=\varepsilon^{-1} u_{\pi}^{-1} u_{\tau}, u_{\tau} u_{\sigma}=u_{\sigma} u_{\tau} .
$$

Clearly, there exists a two-cocycle $c_{1} \in Z^{2}\left(G_{1}, \mathbb{C}^{\times}\right)$, cohomologous to $\beta$, such that $u_{g_{1}} u_{g_{2}}=c_{1}\left(g_{1}, g_{2}\right) u_{g_{1} g_{2}}$ for all $g_{1}, g_{2} \in G_{1}$ and moreover the class $\left[c_{1}\right]$ is determined by (3).

Now, by Karpilovsky [16, Proposition 10.6.1] there exist a two-cocycle $c_{0} \in$ $Z^{2}\left(G, \mathbb{C}^{\times}\right)$and elements $\left\{u_{g}\right\}_{g \in \Phi}$ in $\mathbb{C}^{c_{0}} G$ that satisfy (3), (11) and $u_{g} u_{\gamma_{i}}=u_{\gamma_{i}} u_{g}$ for $g \in\{\pi, \sigma, \tau\}$. We denote this set of equations by $E_{G}$. As in the previous cases, also here, $\left[c_{0}\right]$ is determined by $E_{G}$, and we say that the cocycle $c_{0}$ is of standard form with respect to $\Phi$.

Now, let $c \in Z^{2}\left(G, \mathbb{C}^{\times}\right)$be a nondegenerate two-cocycle, and consider the algebra $\mathbb{C}^{c} G=\bigoplus_{g \in G} \mathbb{C} u_{g}$. Let $\Phi^{(0)}=\left\{\pi^{(0)}, \sigma^{(0)}, \tau^{(0)}, \gamma_{1}^{(0)}, \ldots, \gamma_{2 m}^{(0)}\right\}=\Phi=$ $\left\{\pi, \sigma, \tau, \gamma_{1}, \ldots, \gamma_{2 m}\right\}$. We may assume (by passing to an equivalent cocycle, if necessary) that $u_{\pi^{(0)}}^{2^{n+1}}=u_{\sigma^{(0)}}^{2^{n}}=u_{\tau^{(0)}}^{2}=1$. Let $\alpha \in \mathbb{C}^{\times}$be determined by the equation $u_{\sigma^{(0)}} u_{\pi^{(0)}} u_{\sigma^{(0)}}^{-1}=\alpha u_{\pi^{(0)}}^{3}$. A straightforward calculation shows that for any $k$ in $\mathbb{N}$,

$$
u_{\sigma^{(0)}}^{k} u_{\pi^{(0)}} u_{\sigma^{(0)}}^{-k}=\alpha^{\frac{3^{k}-1}{2}} u_{\pi^{(0)}}^{3^{k}}, 2^{k} \mid \frac{3^{2^{k}-1}}{2} \text { and } 2^{k+1} \nmid \frac{3^{2^{k}-1}}{2} .
$$

We claim that $\alpha$ is a primitive $2^{n+1}$-th root of unity. To see this note that $\sigma^{(0)}$ and $\left(\pi^{(0)}\right)^{2^{n}}$ commute and therefore, $u_{\sigma^{(0)}} u_{\pi^{(0)}}^{2^{n}} u_{\sigma^{(0)}}^{-1}= \pm u_{\pi^{(0)}}^{2^{n}}$. We need to show that $u_{\sigma^{(0)}} u_{\pi^{(0)}}^{2^{n}} u_{\sigma^{(0)}}^{-1}=-u_{\pi^{(0)}}^{2^{n}}$. If not we have

$$
u_{\pi^{(0)}}^{2^{n}}=\left(u_{\sigma^{(0)}} u_{\pi^{(0)}} u_{\sigma^{(0)}}^{-1}\right)^{2^{n}}=\left(\alpha u_{\pi^{(0)}}^{3}\right)^{2^{n}}=\alpha^{2^{n}} u_{\pi^{(0)}}^{2^{n}},
$$

that is, $\alpha^{2^{n}}=1$. Then, by (41) we have

$$
u_{\sigma^{(0)}}^{2^{n-1}} u_{\pi^{(0)}} u_{\sigma^{(0)}}^{-2^{n-1}}=\alpha^{\frac{3^{2^{n-1}}-1}{2}} u_{\pi^{(0)}}^{3^{2^{n-1}}}=u_{\pi^{(0)}}
$$

and hence $u_{\sigma^{(0)}}^{2^{n-1}}$ is in the center of $\mathbb{C}^{c} G$. This contradicts the nondegeneracy of the cocycle $c$ and the claim is proved. It follows that if we replace $\pi^{(0)}$ by a suitable odd power of $\pi^{(0)}$ (that is, $\pi^{(1)}=\pi^{(0)^{l}}, l$ is odd) and leave the other generators unchanged, we obtain a generating set $\Phi^{(1)}=\left\{\pi^{(1)}, \sigma^{(1)}, \tau^{(1)} \gamma_{1}^{(1)}, \ldots, \gamma_{2 m}^{(1)}\right\}$ of $G$, equivalent to $\Phi$, such that $u_{\sigma^{(1)}} u_{\pi^{(1)}} u_{\sigma^{(1)}}^{-1}=\varepsilon u_{\pi^{(1)}}^{3}$.

Next, since $\sigma^{(1)}$ and $\tau^{(1)}$ commute and $\tau^{(1)}$ is of order 2 , we have that $\left(u_{\sigma^{(1)}}, u_{\tau^{(1)}}\right)$ $= \pm 1$. If the commutator is -1 , we put $\tau^{(2)}=\tau^{(1)} \pi^{(1)^{2^{n}}}$ and $\sigma^{(2)}=\sigma^{(1)}$. Otherwise we leave both unchanged and, in either case, get an equivalent generating set $\Phi^{(2)}$ with $\left(u_{\sigma^{(2)}}, u_{\tau^{(2)}}\right)=1$.

Let $\beta \in \mathbb{C}^{\times}$be determined by the equation $u_{\tau^{(2)}} u_{\pi^{(2)}} u_{\tau^{(2)}}^{-1}=\beta u_{\pi^{(2)}}^{-1}$. Then

$$
u_{\sigma^{(2)}} u_{\tau^{(2)}} u_{\pi^{(2)}} u_{\tau^{(2)}}^{-1} u_{\sigma^{(2)}}^{-1}=\beta \varepsilon^{-1} u_{\pi^{(2)}}^{-3} \text {, and } u_{\tau^{(2)}} u_{\sigma^{(2)}} u_{\pi^{(2)}} u_{\sigma^{(2)}}^{-1} u_{\tau^{(2)}}^{-1}=\varepsilon \beta^{3} u_{\pi^{(2)}}^{-3}
$$

and by equality of the left hand sides we have that $\beta= \pm \varepsilon^{-1}$. Now put $\tau^{(3)}=$ $\tau^{(2)}\left(\sigma^{(2)}\right)^{2^{n-1}}$ if $\beta=-\varepsilon^{-1}$ and $\tau^{(3)}=\tau^{(2)}$ otherwise. Leaving the other generators unchanged we obtain an equivalent set of generators $\Phi^{(3)}$ such that the elements 
$u_{\tau^{(3)}}, u_{\pi^{(3)}} \in \mathbb{C}^{c} G$ satisfy $u_{\tau^{(3)}} u_{\pi^{(3)}} u_{\tau^{(3)}}^{-1}=-\beta u_{\pi^{(3)}}^{-1}=\varepsilon^{-1} u_{\pi^{(3)}}^{-1}$. Thus we have shown there exist $\left\{u_{g}\right\}_{g \in\left\{\pi^{(3)}, \sigma^{(3)}, \tau^{(3)}\right\}}$ in $\mathbb{C}^{c} G_{1}$ that satisfy (3).

Next, we may proceed as in Aljadeff, et al. ([2, Proposition 13]) to get a generating set $\Phi^{(4)}$ of $G$, equivalent to $\Phi$, such that (a) (3) is satisfied, (b) $\left(u_{\gamma_{2 k-1}^{(4)}}, u_{\gamma_{2 k}^{(4)}}\right)=$ \pm 1 for all $1 \leq k \leq m$ and (c) all other commutators of the $u_{\gamma_{i}^{(4)}}$ 's are trivial.

The next step is to add the condition $u_{g} u_{\gamma_{i}}=u_{\gamma_{i}} u_{g}$ for $g \in\{\pi, \sigma, \tau\}$ :

For each $\gamma_{i}^{(4)} \in\left\{\gamma_{1}^{(4)}, \ldots, \gamma_{2 m}^{(4)}\right\}$, if $\left(u_{\gamma_{i}^{(4)}}, u_{\pi^{(4)}}\right)=-1$, then put $\gamma_{i}^{(5)}=$ $\gamma_{i}^{(4)}\left(\sigma^{(4)}\right)^{2^{n-1}}$; otherwise leave $\gamma_{i}^{(4)}$ unchanged. Leaving $\pi^{(4)}, \sigma^{(4)}, \tau^{(4)}$ unchanged we obtain a generating set $\Phi^{(5)}$ such that $\left(u_{\gamma_{i}^{(5)}}, u_{\pi^{(5)}}\right)=1$ for all $i$. Next, if $\left(u_{\gamma_{i}^{(5)}}, u_{\sigma^{(5)}}\right)=-1$ put $\gamma_{i}^{(6)}=\gamma_{i}^{(5)}\left(\pi^{(5)}\right)^{2^{n}}$; otherwise leave $\gamma_{i}^{(5)}$ unchanged. We obtain a generating set $\Phi^{(6)}$ such that $\left(u_{\gamma_{i}^{(6)}}, u_{\sigma^{(6)}}\right)=1$ for all $i$.

At this point we have for each $1 \leq k \leq m,\left(u_{\gamma_{2 k-1}^{(6)}}, u_{\gamma_{2 k}^{(6)}}\right)= \pm 1$. In fact we $\underline{\text { claim }}$ that $\left(u_{\gamma_{2 k-1}^{(6)}}, u_{\gamma_{2 k}^{(6)}}\right)=-1$. If not, by the preceding steps we have that the element $u_{\gamma_{2 k-1}^{(6)}}$ centralizes all the $u_{\gamma_{i}^{(6)}}$ 's as well as $u_{\pi^{(6)}}$ and $u_{\sigma^{(6)}}$. It follows that $u_{\gamma_{2 k-1}^{(6)}}$ does not centralize $u_{\tau^{(6)}}$, for otherwise we get a contradiction to the nondegeneracy of the two-cocycle $c$. A similar argument shows that $u_{\gamma_{2 k}^{(6)}}$ does not centralize $u_{\tau^{(6)}}$, but then the product $u_{\gamma_{2 k-1}^{(6)}} u_{\gamma_{2 k}^{(6)}}$ is central, a contradiction. This proves the claim. We refer to the pair of elements $\gamma_{2 k-1}, \gamma_{2 k}$ as partners. We proceed now as follows: If $\left(u_{\gamma_{1}^{(6)}}, u_{\tau^{(6)}}\right)=-1$, put $\tau^{(7)}=\tau^{(6)} \gamma_{2}^{(6)}$ (i.e. multiply $\tau^{(6)}$ by the partner of $\gamma_{1}^{(6)}$ ), and leave $u_{\gamma_{1}^{(6)}}$ and all the other generators unchanged. We continue in a similar way with $\gamma_{2}^{(6)}, \gamma_{3}^{(6)}, \ldots, \gamma_{2 m}^{(6)}$.

We now have a generating set $\Phi^{(r)}$ (some $r$ ), equivalent to $\Phi$, such that $\left\{u_{g}\right\}_{g \in \Phi^{(r)}}$ in $\mathbb{C}^{c} G$ satisfy (a) equations (3), (b) $\left(u_{\gamma_{2 k-1}^{(r)}}, u_{\gamma_{2 k}^{(r)}}\right)=-1$ for all $1 \leq k \leq m$, (c) all other commutators of the $u_{\gamma_{i}^{(r)}}$ 's are trivial and $(\mathrm{d}) u_{\gamma_{i}^{(r)}}$ centralizes the subalgebra $\mathbb{C}\left(u_{\pi^{(r)}}, u_{\sigma^{(r)}}, u_{\tau^{(r)}}\right)$ for all $i$. It follows that the cocycle $c$ is of standard form with respect to $\Phi^{(r)}$.

(IV) In the last step we consider the group $G=C_{4} \rtimes\left(C_{2} \times C_{2}\right) \times C_{2} \times C_{2} \times \ldots \times$ $C_{2} \times C_{2}$ with a set of generators $\Phi=\left\{\pi, \sigma, \tau, \gamma_{1}, \ldots, \gamma_{2 m}\right\}$, where $\sigma \pi \sigma^{-1}=\pi^{3}$ and $\tau \pi=\pi \tau$. We write $G=G_{1} \times G_{2}$, where $G_{1}=C_{4} \rtimes\left(C_{2} \times C_{2}\right)$ and $G_{2}$ is elementary abelian of rank $2 m$.

As in the previous case, a construction of a $K$-central division algebra of the form $K^{\beta} G_{1}$, analogous to that in Natapov [17, yields a $4 \times 4$ matrix algebra isomorphic to $\mathbb{C}^{c_{1}} G_{1}$ with a basis $\left\{u_{g}\right\}_{g \in G_{1}}$ such that $u_{\pi^{i} \sigma^{j} \tau^{k}}=u_{\pi}^{i} u_{\sigma}^{j} u_{\tau}^{k}$ and

$$
u_{\pi}^{4}=u_{\sigma}^{2}=u_{\tau}^{2}=1, u_{\sigma} u_{\pi}=\varepsilon u_{\pi}^{3} u_{\sigma}, u_{\tau} u_{\pi}=-u_{\pi} u_{\tau}, u_{\tau} u_{\sigma}=u_{\sigma} u_{\tau}
$$

where $\varepsilon$ is a fourth root of unity. Clearly, the class $\left[c_{1}\right]$ is determined by (5).

Now, by Karpilovsky [16, Proposition 10.6.1] there exist a two-cocycle $c_{0} \in$ $Z^{2}\left(G, \mathbb{C}^{\times}\right)$and elements $\left\{u_{g}\right\}_{g \in \Phi}$ in $\mathbb{C}^{c_{0}} G$ that satisfy (5), (11), and $u_{g} u_{\gamma_{i}}=u_{\gamma_{i}} u_{g}$ for $g \in\{\pi, \sigma, \tau\}$. We denote these equations by $E_{G}$. Note that $\left[c_{0}\right]$ is determined by $E_{G}$. As usual we will say that the cocycle $c_{0}$ is of standard form with respect to $\Phi$. 
Now, let $c \in Z^{2}\left(G, \mathbb{C}^{\times}\right)$be a nondegenerate two-cocycle and write $\mathbb{C}^{c} G=$ $\bigoplus_{g \in G} \mathbb{C} u_{g}$. As in the previous steps, put $\Phi^{(0)}=\left\{\pi^{(0)}, \sigma^{(0)}, \tau^{(0)}, \gamma_{1}^{(0)}, \ldots, \gamma_{2 m}^{(0)}\right\}=$ $\Phi=\left\{\pi, \sigma, \tau, \gamma_{1}, \ldots, \gamma_{2 m}\right\}$. We may assume (by passing to an equivalent cocycle, if necessary) that $u_{\pi^{(0)}}^{4}=u_{\sigma^{(0)}}^{2}=u_{\tau^{(0)}}^{2}=1$.

We claim that the element $\alpha \in \mathbb{C}^{\times}$determined by the equation $u_{\sigma^{(0)}} u_{\pi^{(0)}} u_{\sigma^{(0)}}^{-1}=$ $\alpha u_{\pi^{(0)}}^{3}$ is a root of unity of order 4 . To see this, note that $\left(u_{g}, u_{\pi^{(0)}}^{2}\right)=1$ for any element $g$ in $G$ of order 2 that commutes with $\pi^{(0)}$. It follows that $\left(u_{\sigma^{(0)}}, u_{\pi^{(0)}}^{2}\right)=-1$, for otherwise $u_{\pi^{(0)}}^{2}$ is contained in the center of the algebra. This proves the claim. Therefore, we may set $\pi^{(1)}=\pi^{(0)^{3}}$ if necessary and we obtain a generating set $\Phi^{(1)}=\left\{\pi^{(1)}, \sigma^{(1)}, \tau^{(1)} \gamma_{1}^{(1)}, \ldots, \gamma_{2 m}^{(1)}\right\}$ of $G$, equivalent to $\Phi$, such that $u_{\sigma^{(1)}} u_{\pi^{(1)}} u_{\sigma^{(1)}}^{-1}=\varepsilon u_{\pi^{(1)}}^{3}$. By the nondegeneracy of $c$ there is an element $h^{(1)} \in$ $\left\{\tau^{(1)}, \gamma_{1}^{(1)}, \ldots, \gamma_{2 m}^{(1)}\right\}$ (these elements generate $C_{G}\left(\pi^{(1)}\right)$, the centralizer of $\pi^{(1)}$ in $G$ ) such that $\left(u_{h^{(1)}}, u_{\pi^{(1)}}\right)=-1$. We set $\tau^{(2)}=h^{(1)}$ and $h^{(2)}=\tau^{(1)}$ and leave the other generators unchanged. Next, arguing as in the previous case, we may assume that $\left(u_{\tau^{(2)}}, u_{\sigma^{(2)}}\right)=1$. Thus we have a generating set $\Phi^{(2)}$ such that $u_{\pi^{(2)}}, u_{\tau^{(2)}}$ and $u_{\sigma^{(2)}}$ satisfy equation (5). Finally, as in the previous case we obtain a generating set $\Phi^{(r)}=\left\{\pi^{(r)}, \sigma^{(r)}, \tau^{(r)}, \gamma_{1}^{(r)}, \ldots, \gamma_{2 m}^{(r)}\right\}$ (some $r$ ), whose elements satisfy (a) $u_{\gamma_{i}^{(r)}}$ centralize the subalgebra $\mathbb{C}\left(u_{\pi^{(r)}}, u_{\sigma^{(r)}}, u_{\tau^{(r)}}\right)$ for all $i,(\mathrm{~b})\left(u_{\gamma_{2 k-1}^{(r)}}, u_{\gamma_{2 k}^{(r)}}\right)=-1$ for all $1 \leq k \leq m$, and (c) all other commutators of the $u_{\gamma_{i}^{(r)}}$ 's are trivial. Therefore $c$ is of standard form with respect to $\Phi^{(r)}$. This completes the proof.

Corollary 19. If $G$ is a group on the list $\Lambda$ and $c$ and $c^{\prime}$ are nondegenerate cocycles on $G$ with values in $\mathbb{C}^{\times}$, then the universal central simple algebras $Q\left(U_{G, c}\right)$ and $Q\left(U_{G, c^{\prime}}\right)$ are isomorphic.

Proof. By Theorem 18 there is an automorphism $\varphi$ of $G$ such that $\varphi(c)$ and $c^{\prime}$ are cohomologous two-cocycles. In particular it follows that $Q\left(U_{G, c}\right)$ and $Q\left(U_{G, c^{\prime}}\right)$ have the same field of definition $\mathbb{Q}(\mu)$ and $\varphi$ induces an automorphism $\varphi_{*}$ of $\mathbb{Q}(\mu)$. Clearly, $\varphi_{*}$ extends to a $\mathbb{Q}$ isomorphism of the free algebras $\Sigma_{c}(\mathbb{Q}(\mu)) \longrightarrow$ $\Sigma_{\varphi(c)}(\mathbb{Q}(\mu))$ by putting $x_{i g} \longmapsto x_{i \varphi(g)}$. Furthermore it induces an isomorphism of the corresponding universal algebras $U_{G, c}=\Sigma_{c}(\mathbb{Q}(\mu)) / T_{c}(\mathbb{Q}(\mu))$ and $U_{G, \varphi(c)}$, and therefore an isomorphism of their central localization, namely the universal central simple $G$-graded algebras.

Corollary 20. Let $G$ be a group of central type and let $c$ be a nondegenerate cocycle on $G$. The universal central simple algebra $Q\left(U_{G, c}\right)$ is a division algebra if and only if $G$ is on the list $\Lambda$.

Proof. This follows from the previous corollary and Proposition 17.

Recall from the introduction that for a group $G$ of central type we let ind $(G)$ denote the maximum over all nondegenerate cocycles $c$ of the indices of the simple algebras $Q\left(U_{G, c}\right)$. We have seen that if $G$ is not on the list, then the universal central simple algebra $Q\left(U_{G}\right)$ is not a division algebra and so the index of $G$ is strictly less than ord $(G)^{1 / 2}$. In fact it is shown in Aljadeff and Natapov 5 that the groups on the list are the only groups responsible for the index of $Q\left(U_{G}\right)$. Here is the precise result. 
Theorem 21. Let $P$ be a p-group of central type and let $Q\left(U_{P, c}\right)$ be the universal central simple $P$-graded algebra for some nondegenerate two-cocycle $c$ on $P$. Then there is a subquotient group $H$ of $P$ on the list $\Lambda$, such that $Q\left(U_{P, c}\right) \cong$ $M_{p^{r}}\left(Q\left(U_{H, \alpha}\right)\right)$, where the cocycle $\alpha$ on $H$ is obtained in a natural way from $c$; namely, there is a subgroup $\hat{H}$ of $P$ such that $\hat{H} / N \cong H$ and $\inf ([\alpha])=\operatorname{res}([c])$ on $\hat{H}$.

Corollary 22. Let $G$ be a central type p-group. Consider all central type groups $H$ that are isomorphic to subquotients of $G$ and are members of $\Lambda$. Then $\operatorname{ind}(G) \leq$ $\sup \left(\operatorname{ord}(H)^{1 / 2}\right)$.

DeMeyer and Janusz prove in [11, Corollary 4] that for an arbitrary group of central type $G$ and a nondegenerate two-cocycle $c \in Z^{2}\left(G, \mathbb{C}^{\times}\right)$, any Sylow $p$ subgroup $G_{p}$ of $G$ is of central type, and furthermore the restriction of $c$ to $G_{p}$ is nondegenerate. Using this result, Geva in his thesis proved that for any subfield $k$ of $\mathbb{C}$ the twisted group algebra $k^{c} G$ is (nongraded) isomorphic to $\bigotimes_{p \mid \operatorname{ord}(G)} k^{c} G_{p}$. It follows that for each prime $p$ dividing the order of $G$ there is a $p$-group $H_{p}$ on the list $\Lambda$ which is isomorphic to a subquotient of $G$ and such that $F^{s} G \cong \bigotimes_{p} M_{p^{r}}\left(F^{\alpha_{p}} H_{p}\right)$. Combining this with the preceding corollary we obtain:

Corollary 23. Let $G$ be a group of central type, and for each prime $p$, let $G_{p}$ be a Sylow p-subgroup of $G$. Consider all central type groups $H_{p}$ that are isomorphic to subquotients of $G_{p}$ and are members of $\Lambda_{p}$. Then $\operatorname{ind}(G) \leq \prod_{p} \sup \left(\operatorname{ord}\left(H_{p}\right)^{1 / 2}\right)$.

It follows from Proposition 17 that for any group $G$ of central type not on the list and any $G$-grading on $M_{n}(\mathbb{C})$, the universal central simple algebra is not a division algebra. That means we can find nonidentity polynomials $p\left(x_{i g}\right)$ and $q\left(x_{i g}\right)$ over the field of definition $\mathbb{Q}(\mu)$ such that $p\left(x_{i g}\right) q\left(x_{i g}\right)$ is a graded identity. In fact there will exist a nonidentity polynomial $p\left(x_{i g}\right)$ over $\mathbb{Q}(\mu)$ such that $p\left(x_{i g}\right)^{r}$ is a graded identity for some positive integer $r$. This is clearly equivalent to saying that under every substitution the value of $p$ in $M_{n}(\mathbb{C})$ is a nilpotent matrix. We will refer to such a polynomial as a nilpotent polynomial.

We present an explicit example of a nilpotent polynomial. We let $S_{3}$ be the permutation group on three letters, and let $C_{6}=\langle z\rangle$ be a cyclic group of order 6 . Let $\sigma=(123)$ and $\tau=(12)$ be generators of $S_{3}$ and define an action of $S_{3}$ on $C_{6}$ by $\tau(z)=z^{-1}$ and $\sigma(z)=z$. We consider the group $G=S_{3} \ltimes C_{6}$. Note that $G$ is not nilpotent and hence not on the list $\Lambda$.

Let $C_{6}^{\vee}=\operatorname{Hom}\left(C_{6}, \mathbb{C}^{\times}\right)=\left\langle\chi_{z}\right\rangle$ denote the dual group of $C_{6}$ and let $\langle$,$\rangle denote$ the usual pairing between $C_{6}$ and $C_{6}^{\vee}$ (i.e. $\langle a, \chi\rangle=\chi(a)$ for all $a \in C_{6}, \chi \in C_{6}^{\vee}$ ). It is well known ([16, Corollary 10.10.2]) that $H^{1}\left(S_{3}, C_{6}^{\vee}\right) \cong H^{2}\left(S_{3} \ltimes C_{6}, \mathbb{C}^{\times}\right)$. The isomorphism may be given by

$$
\pi \mapsto c: c\left(h_{1} a_{1}, h_{2} a_{2}\right)=\left\langle h_{2} \cdot a_{1}, \pi\left(h_{2}\right)\right\rangle,
$$

for all $h_{1} a_{1}, h_{2} a_{2} \in S_{3} \ltimes C_{6}$. Note that the restriction of $c$ on $S_{3}$, as well as on $C_{6}$, is trivial.

Moreover, it is shown in Etingof and Gelaki [12 that there exists a bijective onecocycle $\pi: S_{3} \rightarrow C_{6}^{\vee}$ and, because of the bijectivity, the corresponding two-cocycle 
$c$ is nondegenerate. In particular this proves that $G$ is of central type (see Etingof and Gelaki [13]). One may define a bijective $\pi$ as follows:

$$
\begin{array}{ll}
\pi(i d)=\chi_{1} & \pi(12)=\chi_{z} \\
\pi(123)=\chi_{z}^{2} & \pi(23)=\chi_{z}^{5} \\
\pi(132)=\chi_{z}^{4} & \pi(13)=\chi_{z}^{3} .
\end{array}
$$

Let $c$ denote the nondegenerate two-cocycle which corresponds to $\pi$. Then the twisted group algebra $\mathbb{C}^{c} G$ is isomorphic to $M_{6}(\mathbb{C})$ or, equivalently, $M_{6}(\mathbb{C})$ is $G$ graded with the class $[c]$. The group $G$ is not on the list $\Lambda$ and hence the corresponding universal algebra $U_{G}$ is not a domain. Consider the subalgebra $\mathbb{C}\left(u_{\sigma}, u_{y}\right)$, where $y=z^{2}$. It is straightforward to verify that the cocycle $c$ satisfies $c(y, \sigma)=\omega^{2}$, where $\omega=e^{\frac{2 \pi i}{3}}$ is a primitive third root of unity in $\mathbb{C}$, and hence $\mathbb{C}\left(u_{\sigma}, u_{y}\right)$ is a symbol algebra. It follows that the corresponding subalgebra $F\left(t_{\sigma} u_{\sigma}, t_{y} u_{y}\right)$ of the universal central simple algebra $Q\left(U_{G, c}\right)=F^{s(c)} G$ is a symbol algebra $(a, b)_{3}$, and by Aljadeff, et al. [3, Lemma 6], $a$ and $b$ are roots of unity. It follows that $F\left(t_{\sigma} u_{\sigma}, t_{y} u_{y}\right)$ is split, that is, isomorphic to $M_{3}(F)$. Thus we know that there exists a degree three nilpotent element in $U_{G, c}$. To construct it we consider the element $u_{\sigma}-u_{y} u_{\sigma}$ in $\mathbb{C}^{c} G$. Note that this is not zero since $u_{\sigma}$ and $u_{y} u_{\sigma}$ are linearly independent over $\mathbb{C}$. Its square

$$
\left(u_{\sigma}-u_{y} u_{\sigma}\right)^{2}=u_{\sigma}^{2}-u_{\sigma} u_{y} u_{\sigma}-u_{y} u_{\sigma}^{2}+\left(u_{y} u_{\sigma}\right)^{2}
$$

is not zero as well, but using $u_{y} u_{\sigma}=\omega^{2} u_{\sigma} u_{y}$ and $u_{y}^{3}=1$ one shows that its third power

$$
\begin{aligned}
\left(u_{\sigma}-u_{y} u_{\sigma}\right)^{3}= & u_{\sigma}^{3}-u_{\sigma}^{2} u_{y} u_{\sigma}-u_{\sigma} u_{y} u_{\sigma}^{2}+u_{\sigma}^{2} u_{y} u_{\sigma} u_{y} u_{\sigma}-u_{y} u_{\sigma}^{3}+u_{y} u_{\sigma}^{2} u_{y} u_{\sigma} \\
& +u_{y} u_{\sigma} u_{y} u_{\sigma}^{2}-\left(u_{y} u_{\sigma}\right)^{3}
\end{aligned}
$$

vanishes.

Now, as mentioned above, $\operatorname{res}_{S_{3}}^{G}(c)=\operatorname{res}_{C_{6}}^{G}(c)=1$; hence we may assume that $u_{g} u_{h}=u_{g h}$ for all $g, h \in S_{3}$ or $g, h \in C_{6}$. In particular, we have $u_{e}=u_{\tau}^{2} u_{y}^{3}$. Also, using the cocycle values as defined in (6), we obtain $u_{y}=\omega u_{\tau} u_{y} u_{\tau} u_{y}^{2}$.

Let $x_{\tau}=x_{1 \tau}, x_{\sigma}=x_{1 \sigma}, x_{y}=x_{1 y}$ be indeterminates in $\Omega=\left\{x_{i g}: i \in \mathbb{N}, g \in G\right\}$. Let the polynomial $f\left(x_{\tau}, x_{\sigma}, x_{y}\right) \in \mathbb{Q}(\omega)\langle\Omega\rangle$ be given by

$$
f\left(x_{\tau}, x_{\sigma}, x_{y}\right)=x_{\sigma} x_{\tau}^{2} x_{y}^{3}-\omega x_{\tau} x_{y} x_{\tau} x_{y}^{2} x_{\sigma} .
$$

It follows that the polynomial $f^{3}\left(x_{\tau}, x_{\sigma}, x_{y}\right)$ is a polynomial identity for $M_{6}(\mathbb{C})$ while $f$ and $f^{2}$ are not.

We end this example by expressing $f^{3}$ explicitly as a $\mathbb{Q}(\omega)$-linear combination of elementary identities:

$$
\begin{aligned}
f^{3}\left(x_{\tau}, x_{\sigma}, x_{y}\right)= & \left(x_{\sigma} x_{\tau}^{2} x_{y}^{3}\right)^{3}-\left(x_{\tau} x_{y} x_{\tau} x_{y}^{2} x_{\sigma}\right)^{3} \\
& +\omega^{2} x_{\tau} x_{y} x_{\tau} x_{y}^{2} x_{\sigma}\left(x_{\sigma} x_{\tau}^{2} x_{y}^{3}\right)^{2}-\omega\left(x_{\sigma} x_{\tau}^{2} x_{y}^{3}\right)^{2} x_{\tau} x_{y} x_{\tau} x_{y}^{2} \\
& +x_{\tau} x_{y} x_{\tau} x_{y}^{2} x_{\sigma}\left(x_{\sigma} x_{\tau}^{2} x_{y}^{3}\right)^{2}-\omega x_{\sigma} x_{\tau}^{2} x_{y}^{3} x_{\tau} x_{y} x_{\tau} x_{y}^{2} x_{\sigma} x_{\sigma} x_{\tau}^{2} x_{y}^{3} \\
& +\omega^{2} x_{\sigma} x_{\tau}^{2} x_{y}^{3}\left(x_{\tau} x_{y} x_{\tau} x_{y}^{2} x_{\sigma}\right)^{2}-\left(x_{\tau} x_{y} x_{\tau} x_{y}^{2} x_{\sigma}\right)^{2} x_{\sigma} x_{\tau}^{2} x_{y}^{3} \\
& +\omega^{2}\left(x_{\tau} x_{y} x_{\tau} x_{y}^{2} x_{\sigma}\right) x_{\sigma} x_{\tau}^{2} x_{y}^{3}\left(x_{\tau} x_{y} x_{\tau} x_{y}^{2} x_{\sigma}\right) \\
& -\omega\left(x_{\tau} x_{y} x_{\tau} x_{y}^{2} x_{\sigma}\right)^{2} x_{\sigma} x_{\tau}^{2} x_{y}^{3}
\end{aligned}
$$


If $G$ is on the list $\Lambda$ and $c$ is a nondegenerate two-cocycle on $G$, we have seen that the algebra $Q\left(U_{G, c}\right)$ is a division algebra of degree $n=\operatorname{ord}(G)^{1 / 2}$. In the next result we calculate the index of $Q\left(U_{G, c}\right) \otimes_{\mathbb{Q}(\mu)} \mathbb{C}$, where $\mathbb{Q}(\mu)$ is the field of definition for the graded identities.

Proposition 24. If $G$ is on the list $\Lambda$ and $c$ is a nondegenerate two-cocycle on $G$, then the index of $Q\left(U_{G, c}\right) \otimes_{\mathbb{Q}(\mu)} \mathbb{C}$ is $n / \operatorname{ord}\left(G^{\prime}\right)$. In particular $Q\left(U_{G, c}\right) \otimes_{\mathbb{Q}(\mu)} \mathbb{C}$ is a division algebra if and only if $G$ is abelian of symmetric type.

Proof. By Aljadeff and Haile [1] (see the discussion at the beginning of section two) the subalgebra $F^{s} G^{\prime}$ of $Q\left(U_{G, c}\right)=F^{s} G$ is a cyclotomic extension of $F$. It follows that the index of $Q\left(U_{G, c}\right) \otimes_{\mathbb{Q}(\mu)} \mathbb{C}$ is at most $n / \operatorname{ord}\left(G^{\prime}\right)$. We proceed to prove the opposite inequality. By Natapov [17, proof of Theorem 6] there is a subfield $K$ of $\mathbb{C}$ containing $\mathbb{Q}(\mu)$ and a nondegenerate cocycle $\beta$ on $G$ with values in $K$ such that the algebra $K^{\beta} G \otimes_{\mathbb{Q}(\mu)} \mathbb{C}$ has index exactly $n / \operatorname{ord}\left(G^{\prime}\right)$. By a specialization argument, it follows that the algebra $Q\left(U_{G, \beta}\right)$ has index at least $n / \operatorname{ord}\left(G^{\prime}\right)$. By Corollary 19, $Q\left(U_{G, \beta}\right)$ is isomorphic to $Q\left(U_{G, c}\right)$ and hence the index of $Q\left(U_{G, c}\right)$ is at least $n / \operatorname{ord}\left(G^{\prime}\right)$.

\section{REFERENCES}

[1] E. Aljadeff, D. Haile, Division algebras with a projective basis, Israel J. Math. 121 (2001) 173-198. MR.1818387 (2002g:16027)

[2] E. Aljadeff, D. Haile, M. Natapov, Projective bases of division algebras and groups of central type, Israel J. Math. 146 (2005) 317-335. MR2151606 (2006c:20005)

[3] E. Aljadeff, D. Haile, M. Natapov, On fine gradings on central simple algebras, Groups, Rings and Group Rings, 1-9, Lecture Notes in Pure and Appl. Math., 248, Taylor and Francis, New York, 2006. MR2226177 (2007m:16030)

[4] E. Aljadeff, C. Kassel, Polynomial identities and noncommutative versal torsors, arXiv:0708.4108, Adv. Math. (2008), doi:10.1016/j.aim.2008.03.014.

[5] E. Aljadeff, M. Natapov, On the universal $G$-graded central simple algebra, Actas del XVI Coloquio Latinoamericano de Álgebra (Colonia, Uruguay, 2005), 115-130, Revista Matemática Iberoamericana, Madrid, 2007.

[6] E. Aljadeff, J. Sonn, Projective Schur algebras of nilpotent type are Brauer equivalent to radical algebras, J. Algebra 220 (1999) 401-414. MR.1717348(2000i:16035)

[7] Yu. Bahturin, V. Drensky, Graded polynomial identities of matrices, Linear Algebra Appl. 357 (2002), 15-34. MR1935223(2003k:16034)

[8] Yu. Bahturin, S. Sehgal, M. Zaicev, Group gradings on associative algebras, J. Algebra 241 (2001) 677-698. MR1843319 (2002h:16067)

[9] Yu. Bahturin, M. Zaicev, Group gradings on matrix algebras. Dedicated to Robert V. Moody. Canad. Math. Bull. 45 (2002), no. 4, 499-508. MR.1941224 (2003h:16046)

[10] F. DeMeyer, E. Ingraham, Separable algebras over commutative rings. Lecture Notes in Mathematics, Vol. 181, Springer-Verlag, Berlin - New York, 1971. MR0280479 (43:6199)

[11] F. DeMeyer, G. Janusz, Finite groups with an irreducible representation of large degree, Math. Z. 108 (1969), 145-153. MR0237629(38:5910)

[12] P. Etingof, S. Gelaki, A method of construction of finite-dimensional triangular semisimple Hopf algebras, Math. Res. Lett. 5 (1998) 551-561. MR1653340(99i:16069)

[13] P. Etingof, S. Gelaki, The classification of triangular semisimple and cosemisimple Hopf algebras over an algebraically closed field, Intern. Math. Res. Notices (2000) no. 5, 223-234. MR:1747109 (2001h:16039)

[14] R. Howlett, I. Isaacs, On groups of central type, Mathematische Zeitschrift 179 (1982) 555569. MR652860 (83j:20020)

[15] I. Isaacs, Character Theory of Finite Groups, Dover Publications, New York, 1994. MR 1280461

[16] G. Karpilovsky, Group representations, Vol. 2, North-Holland, Amsterdam, 1993. MR.1215935 (94f:20001) 
[17] M. Natapov, Projective bases of division algebras and groups of central type. II, Israel J. Math. 164 (2008) 61-73. MR2391140 (2009a:16032)

[18] I. Reiner, Maximal Orders, Academic Press, London, 1975. MR,1972204 (2004c:16026)

Department of Mathematics, Technion-Israel Institute of Technology, Haifa 32000 , ISRAEL

E-mail address: aljadeff@tx.technion.ac.il

Department of Mathematics, Indiana University, Bloomington, Indiana 47405

E-mail address: haile@indiana.edu

Department of Mathematics, Indiana University, Bloomington, Indiana 47405

E-mail address: mnatapov@indiana.edu

Current address: Department of Mathematics, Technion-Israel Institute of Technology, Haifa 32000 , Israel

E-mail address: natapov@tx.technion.ac.il 